\title{
Norois
}

Environnement, aménagement, société

$191 \mid 2004 / 2$

Les types de temps

\section{L'îlot de chaleur urbain parisien selon les types de temps}

Weather types and Urban Heat Island in Paris

\section{Olivier Cantat}

\section{(2) OpenEdition \\ 1 Journals}

Édition électronique

URL : https://journals.openedition.org/norois/1373

DOI : $10.4000 /$ norois. 1373

ISBN : 978-2-7535-1539-0

ISSN : 1760-8546

\section{Éditeur}

Presses universitaires de Rennes

\section{Édition imprimée}

Date de publication : 1 mars 2004

Pagination : 75-102

ISBN : 978-2-86847-977-8

ISSN : 0029-182X

\section{Référence électronique}

Olivier Cantat, «L'îlot de chaleur urbain parisien selon les types de temps », Norois [En ligne], 191 |

2004/2, mis en ligne le 10 septembre 2008, consulté le 14 janvier 2022. URL : http://

journals.openedition.org/norois/1373 ; DOI : https://doi.org/10.4000/norois.1373

Ce document a été généré automatiquement le 14 janvier 2022.

(c) Tous droits réservés 


\title{
L'îlot de chaleur urbain parisien selon les types de temps
}

Weather types and Urban Heat Island in Paris

\author{
Olivier Cantat
}

\section{Introduction}

Dans les grandes agglomérations, l'extension des zones bâties et les rejets de pollution atmosphérique engendrent des modifications locales du bilan d'énergie. L'apparition d'un îlot de chaleur urbain (ICU), sorte de dôme d'air plus chaud couvrant la ville, est la manifestation climatique la plus concrète de la présence et des activités de la ville. L'ICU est la résultante d'un ensemble de facteurs, parfois antagonistes, agissant à différentes échelles. Ses caractères reflètent la multitude des combinaisons possibles entre les conditions climatiques régionales, le contexte saisonnier, les caractéristiques propres à chaque ville (cadre physique, forme et densité du bâti, nature des matériaux de construction, activités industrielles...) et la diversité des types de temps. De ce fait, chaque agglomération possède sa propre identité thermique, elle-même très variable dans l'espace comme dans le temps. La multiplication de vastes mégalopoles depuis une cinquantaine d'années a donné lieu à de très nombreuses publications sur l'ICU, avec notamment les contributions de Chandler (1965) sur Londres, Bornstein (1968) et Oke (1973) en Amérique du Nord, Dettwiller (1970) et Escourrou (1986a) sur Paris.

2 Le but de cet article n'est pas de proposer une approche exhaustive de l'îlot de chaleur parisien - qui a fait d'ailleurs l'objet de nombreuses études directes (Dettwiller 1970, Escourrou 1988, Cantat 1989...) ou indirectes (Maurain 1947, Grisollet 1958, Pédelaborde 1958, Requillard 1961, Bessemoulin 1980, Calvet 1984...) - mais de s'interroger sur les relations parfois complexes entre cette anomalie thermique et la diversité des types de temps qui se succèdent aux latitudes moyennes océaniques.

3 Après avoir présenté succinctement le cadre géoclimatique de l'étude et précisé le concept essentiel de type de temps ( $1^{\text {re }}$ partie), nous évoquerons les moyens d'analyse de l'ICU et sa genèse ( $2^{\mathrm{e}}$ partie), puis ses fluctuations dans le temps et dans l'espace ( $3^{\mathrm{e}}$ 
et $4^{\mathrm{e}}$ partie). Enfin, la structure verticale de l'atmosphère nous permettra de mieux appréhender les mécanismes de formation de cette anomalie thermique propre aux espaces urbains (5e partie). Une ouverture sur la modélisation et la prévision de ce phénomène local discontinu et mouvant conclura notre propos.

\section{Cadre géoclimatique et interrelations type de temps / îlot de chaleur urbain}

\section{Un contexte régional peu compatible avec l'établissement de topoclimats}

Le cas de Paris est représentatif d'une très vaste agglomération (photo 1) dont le climat régional est plutôt défavorable à l'établissement des climats locaux (topoclimats). En effet, la situation géographique de la région parisienne impose un climat océanique, marqué par la grande variabilité des types de circulation, avec prédominance des conditions cycloniques turbulentes (Pédelaborde 1958). Cette agitation ne favorise pas l'expression des facteurs géographiques locaux sur les basses couches de l'atmosphère. A une échelle locale, cependant, le site de l'agglomération corrige un peu la situation géographique avec une urbanisation qui s'est préférentiellement "coulée dans les vallées avant de gagner les plateaux » (Bastié, 1984). Au total, l'éloignement des côtes et la position d'abri en vallée de la Seine, expliquent les vents relativement faibles qui permettent aux influences urbaines de s'établir.

Photo 1 : Le centre de la région parisienne : une agglomération à perte de vue...

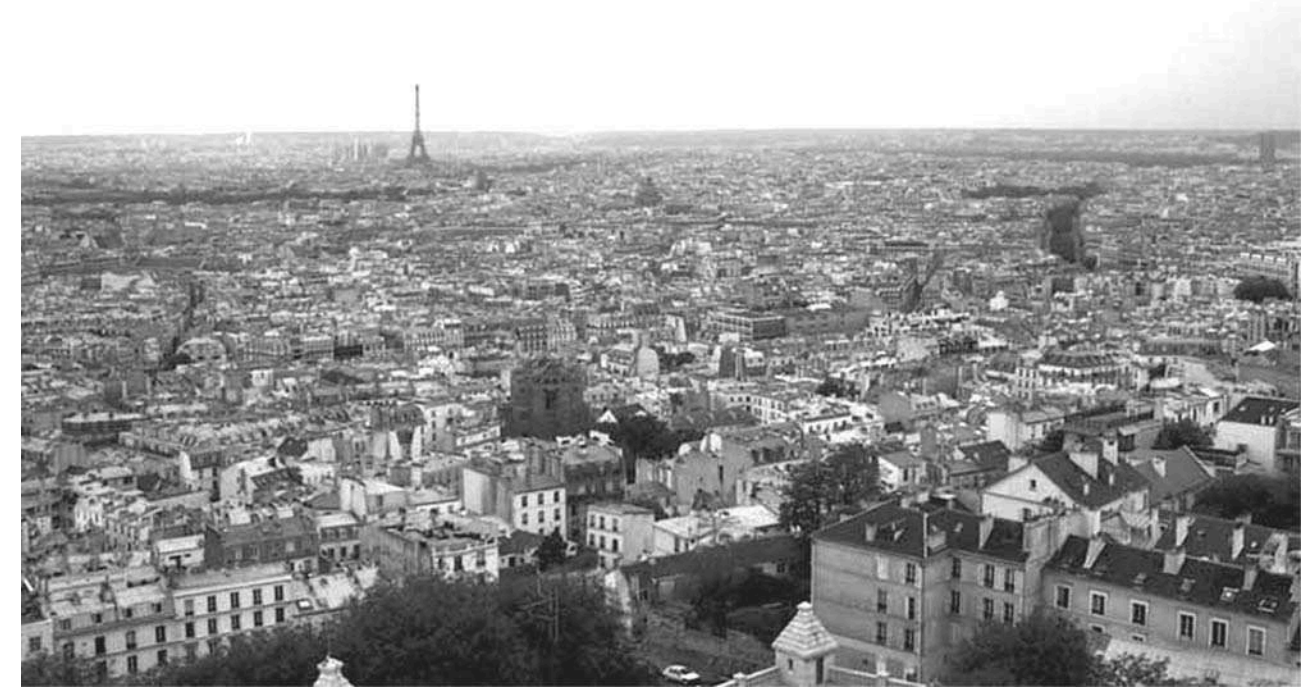

Vue vers le S-W de la capitale, à partir du dôme du Sacré Cœur

$(\mathrm{OC}, 2003)$ 


\section{Définition du concept de type de temps : un préalable indispensable}

Le type de temps peut être défini comme une combinaison homogène plus ou moins durable d'éléments atmosphériques sur un espace d'échelle régionale à locale. Cette définition correspond à l'élargissement dans l'espace et dans le temps de la notion de temps proposée en 1934 par Albert Baldit : «le temps (...) est l'ensemble des valeurs qui, à un moment donné et en un lieu déterminé, caractérisent l'état de l'atmosphère » (Piery).

6 Cette définition très synthétique du type de temps est nécessaire car il y a parfois confusion avec le concept de type de circulation qui s'applique, lui, à des proportions d'espace et de temps plus importantes. Dans la pratique, il y a imbrication de ces deux échelles. Concrètement, un type de circulation peut affecter toute l'Europe occidentale et perdurer parfois plusieurs semaines (cf. flux d'ouest perturbé à la saison froide), ce qui n'empêche pas la succession dans le temps (durée) et la juxtaposition dans l'espace de plusieurs types de temps en relation avec les facteurs géographiques comme la topographie et le mode d'occupation du sol. Ces ambiguités de langage ont donné lieu à de nombreuses discussions au sein de la communauté des géoclimatologues, notamment en 1973 lors de la Commission de Grenoble et, trente ans plus tard, lors de ces journées de la Commission « Climat et Société » de Rennes.

7 Toutefois, la vision différenciée des types de circulation et des types de temps n'exclut pas la réunion de ces deux concepts dans la recherche des facteurs explicatifs structurants de l'atmosphère. "Tout phénomène d'un certain ordre de grandeur est un ensemble de phénomènes d'ordre de grandeur immédiatement inférieur, et compte parmi ses composantes explicatives un phénomène d'ordre de grandeur immédiatement supérieur » (Durand-Dastès 1973).

8 Dans une approche géographique de l'influence des types de temps sur l'îlot de chaleur urbain parisien, le type de temps sera défini comme l'unité temporelle de base durant laquelle agit une combinaison d'éléments atmosphériques sensiblement identiques et produisant des effets pratiquement semblables sur un espace donné. Sous notre climat, cette unité temporelle de base peut varier de quelques heures seulement à plusieurs jours. Si le temps est clair et calme, les facteurs géographiques locaux peuvent s'exprimer pleinement et déterminer des topoclimats très différenciés. Quand ce type de temps perdure, l'ICU s'affirme davantage, au point d'apparaitre encore plus franchement sur la moyenne d'une décade, voire d'un mois.

\section{ICU et type de temps : causes et effets, relations et interrelations...}

\section{L'ICU peut-être considéré comme élément dépendant des types de temps :}

9 A Paris, l'influence de l'urbanisation sur les températures est suffisante pour apparaître dans les statistiques et sur les cartes des atlas climatologiques mais ces données «moyennes » masquent souvent des configurations thermiques encore plus tranchées, étroitement dépendantes des types de temps. À l'échelle journalière, l'écart des températures minimales entre le centre de Paris et le milieu le plus froid de la campagne peut dépasser quelquefois $10^{\circ} \mathrm{C}$ (fig. 1). 
Fig.1 : L'influence des types de temps sur l'îlot de chaleur urbain parisien

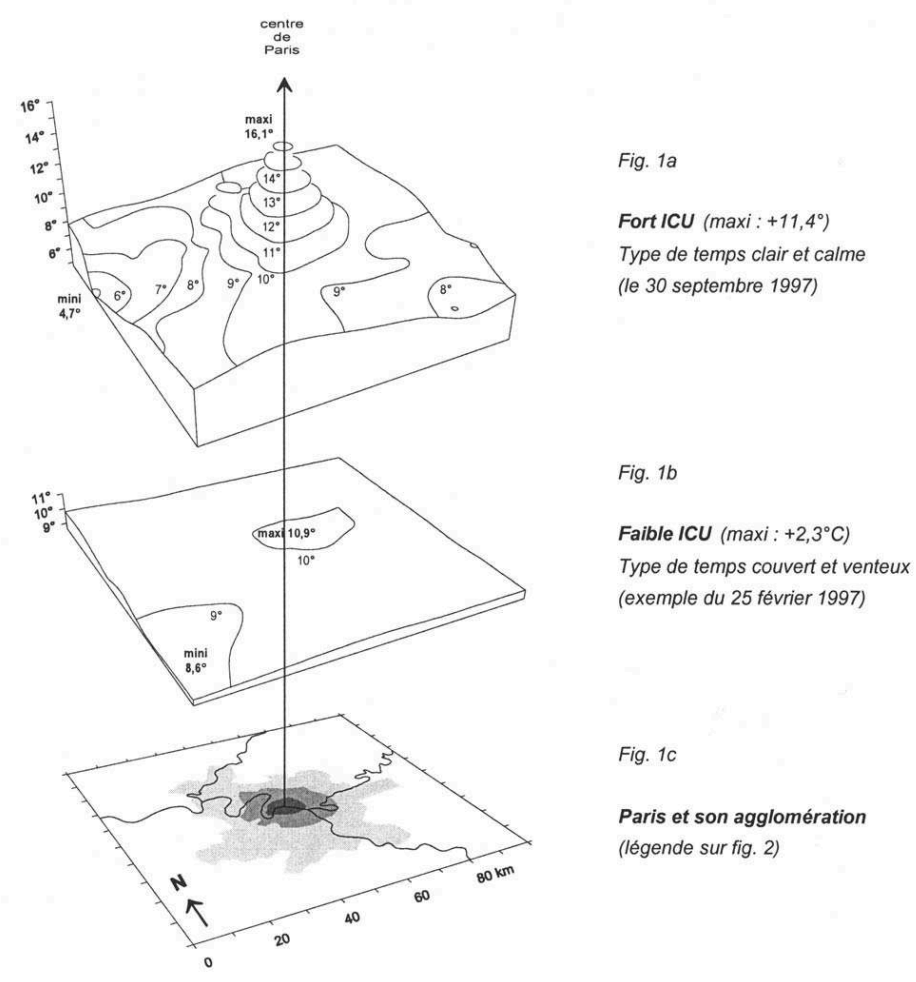

L'ICU peut être étudié comme élément agissant sur le type de temps :

10 Un type de circulation détermine donc un type de temps mais il est fréquent que plusieurs types de temps coexistent à l'échelle régionale, voire locale. Les caractères originaux du milieu urbain conduisent parfois à l'individualisation d'un type de temps qui diffère de la campagne environnante : le cas le plus flagrant pour le citadin parisien est constitué par la disparition quasi totale des brouillards de rayonnement nocturne au centre de l'agglomération et l'élévation de la base des nuages bas (Escourrou 1983, Cantat 1993). Une amélioration sensible des conditions de visibilité est également perceptible en banlieue parisienne (Barrot 1970, Le Guischer et Tschirharrt 1981, Janicot 1984, Cantat 1984). Par temps faiblement instable, le ciel moins «chargé » sur Paris occasionne des précipitations fines plus atténuées et moins durables. Au contraire, par forte instabilité, les nuages cumuliformes apparaissent plus développés, renforçant l'intensité des averses au centre et sous le vent de l'agglomération (Calvet 1980, Escourrou 1980, Cantat 1988a). Ces modifications locales du type de temps régional découlent pour l'essentiel de la formation d'un îlot de chaleur urbain qui "entraîne une hétérogénéité, dans l'espace, de la répartition de divers paramètres météorologiques liés à la température des très basses couches : gradient thermique vertical, humidité, nébulosité, brumes et brouillards, etc.» (Dettwiller 1970). Si l'approche de l'ICU comme élément agissant sur le type de temps n'est pas l'objet de notre analyse, cette dimension sera toutefois fréquemment sous-jacente étant donné les interdépendances entre "îlot de chaleur» urbain, types de temps et situation synoptique. 


\section{Moyens d'analyse et facteurs explicatifs de l'îlot de chaleur parisien}

\section{Un réseau de stations dense permettant de traduire l'allure générale de l'ICU}

En région parisienne, la densité du réseau de mesures (fig. 2) permet de préciser les caractéristiques d'intensité, de durée, de fréquence, de forme et d'étendue de cette « anomalie » thermique.

Fig. 2 : Densité actuelle du réseau de mesures thermométriques et principales auréoles urbaines

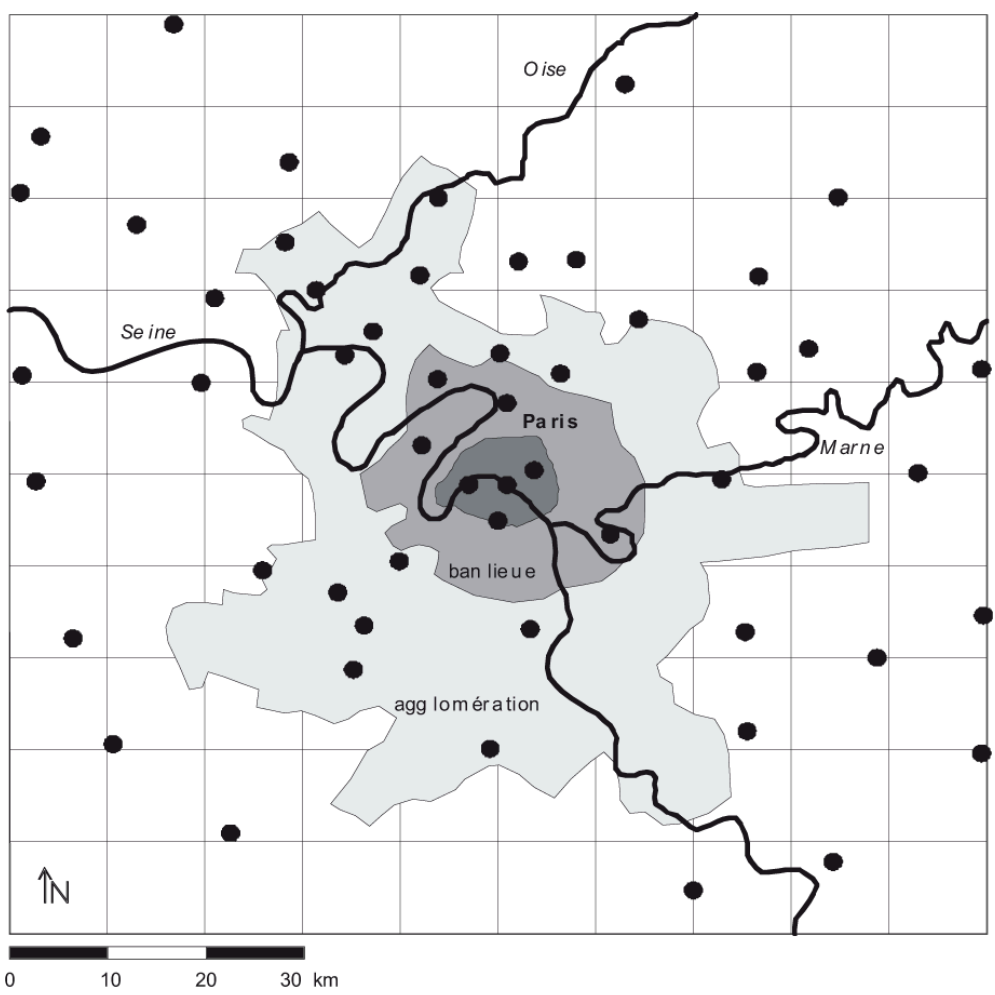

Nota : la schématisation des principales auréoles urbaines a été effectuée par numérisation de cartes I.G.N au 1/100.000 de la région parisienne. Les surfaces mentionnées dans le tableau 1 donnent un ordre de grandeur approximatif des principales aires ainsi définies.

Actuellement, 55 postes thermiques couvrent les $10.000 \mathrm{~km}^{2}$ de notre aire d'étude (tab. 1). L'agglomération parisienne $\left(2.530 \mathrm{~km}^{2}\right.$ environ) compte 24 postes, soit en moyenne près de 1 station par maille carrée de 10 kilomètres de côté, ce qui constitue une densité très élevée comparativement aux autres régions françaises. Le centre de l'agglomération (Paris + proche banlieue) est pourvu de 9 stations, dont celle référence du square St-Jacques, au cœur de la capitale mais dont les données ne sont malheureusement plus accessibles depuis septembre 2001. La référence rurale sera généralement fournie par la station de Melun, $37 \mathrm{~km}$ au sud-est de Paris (aérodrome de Melun-Villaroche). Ces deux références urbaine et rurale sont localisées sur la figure 3. 
Tab. 1 :Densité du réseau thermométrique en 1999 et auréoles urbaines

\begin{tabular}{|l|c|c|c|}
\cline { 2 - 4 } \multicolumn{1}{c|}{} & $\begin{array}{c}\text { surface } \\
\mathrm{km}^{2}\end{array}$ & $\begin{array}{c}\text { postes } \mathrm{T}^{\circ} \\
\mathrm{nb} \text { total }\end{array}$ & $\begin{array}{c}\text { densité } \\
\text { postes } / 100 \mathrm{~km}^{2}\end{array}$ \\
\hline Paris ville & 93 & 4 & 4,30 \\
\hline banlieue & 467 & 5 & 1,07 \\
\hline agglomération & 1970 & 15 & 0,76 \\
\hline Total agglo. & $\mathbf{2 5 3 0}$ & $\mathbf{2 4}$ & $\mathbf{0 , 9 5}$ \\
\hline \multicolumn{4}{|c|}{} \\
\hline "campagne" & $\mathbf{7 4 7 0}$ & $\mathbf{3 1}$ & $\mathbf{0 , 4 1}$ \\
\hline & $\mathbf{1 0 0 0 0}$ & $\mathbf{5 5}$ & $\mathbf{0 , 5 5}$ \\
\hline
\end{tabular}

Fig. 3 : Variations mensuelles des fluctuations horaires de l'ICU parisien entre 1951 et 1980 (thermo-isoplèthes entre les valeurs tri-horaires reconstituées de Paris-St-Jacques et Melun)

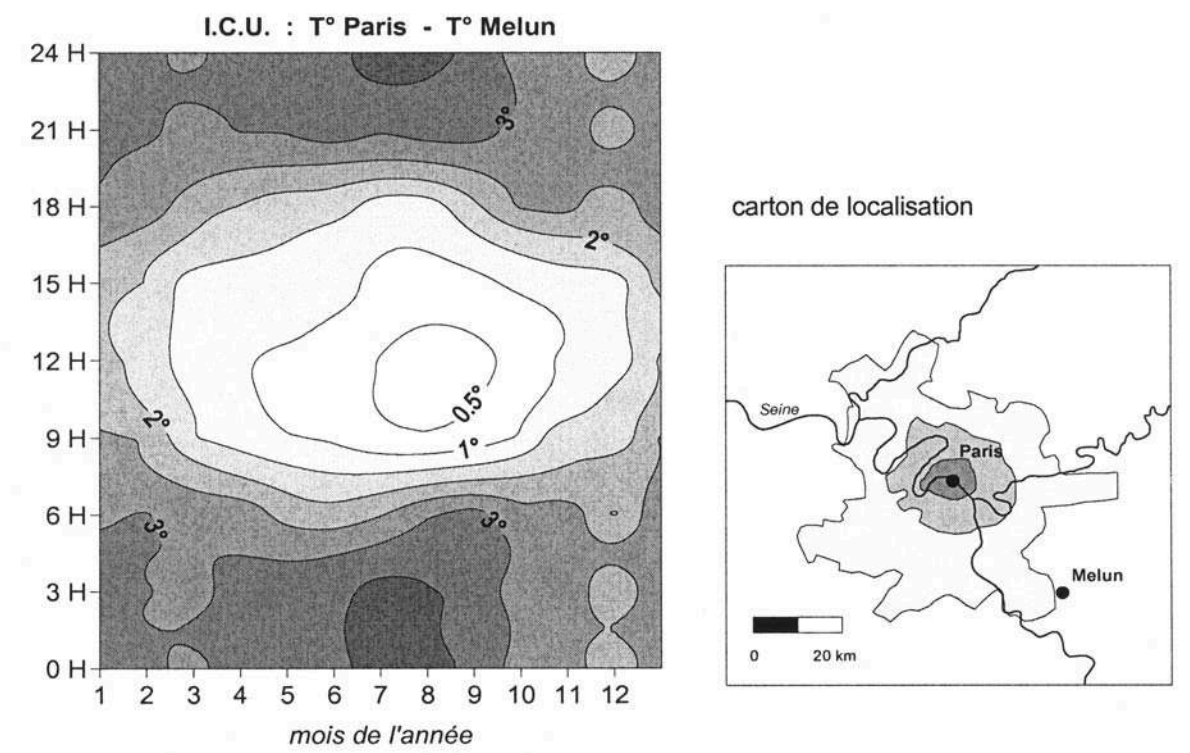

13 Cette haute densité et cette répartition des stations permettent une approche spatiale du climat urbain de Paris, notamment par la production de cartes en isolignes d'une grande lisibilité pour matérialiser l'ICU. La validité des isothermes est fonction avant tout de la densité des mesures et de la représentativité géographique des sites : pour garantir la cohérence des mesures, les stations retenues pour l'étude sont toutes situées dans des parcs ou sur des espaces végétalisés. Les rares stations à proximité immédiate de sources artificielles de chaleur (béton, parking, bâtiment...) ont été supprimées. Cette précaution permet d'assurer que l'ICU n'est pas simplement dû à l'environnement immédiat de l'abri météorologique, mais bien révélateur d'une anomalie locale du champ thermique régional.

14 Autre problème, l'interpolation spatiale lisse la diversité réelle de la température à échelle très fine, liée à la topographie et aux modes d'occupation du sol... Dans notre cas, cette précision de détail n'était pas requise pour appréhender les relations entre 
îlot de chaleur et types de temps. Notons que la modélisation fine de l'ICU fait l'objet de nombreuses recherches, dont notamment les travaux sur la métropole lilloise (Charabi et al., 2002). Les cartes proposées dans la $4^{\text {ème }}$ partie devront donc s'apprécier à moyenne échelle, les isothermes n'ayant d'autre vocation que de schématiser l'allure générale de l'ICU.

Cette situation très favorable pour analyser la géographie actuelle du climat urbain parisien résulte d'un effort de création de postes par Météo-France, principalement durant la seconde moitié des années 1990 (tab.2). Si la partie centrale de l'agglomération n'a pas connu d'augmentation majeure du nombre de postes thermiques entre les décennies 1961-1970 et 1991-2000 (de 4 à 7 pour le groupe central Paris, Hauts-de-Seine, Seine-St-Denis et Val-de-Marne), en revanche les départements périphériques ont enregistré une hausse significative (de 13 à 37 pour l'ensemble Vald'Oise, Seine-et-Marne, Yvelines et Essonne). Cette densité permet de percevoir les variations spatiales de l'ICU avec beaucoup plus de précisions qu'autrefois et d'envisager les relations centre-banlieue-campagne.

Tab. 2 : Evolution du nombre de postes thermiques entre 1961 et 2000

\begin{tabular}{|c|c|c|c|c|c|c|}
\hline & \multicolumn{4}{|c|}{ évolution inter décennale } & \multicolumn{2}{|c|}{ "actuelle" } \\
\hline & 1961-1970 & $1971-1980$ & 1981-1990 & $1991-2000$ & $1996-2000$ & 2000 \\
\hline $\begin{array}{l}\text { départements du centre et proche } \\
\text { banlieue }(75,92,93,94)\end{array}$ & 4 & 4 & 4 & 3 & 6 & 7 \\
\hline $\begin{array}{l}\text { départements de banlieue lointaine } \\
\text { et campagne }(77,78,91,95)\end{array}$ & 13 & 17 & 22 & 37 & 59 & 64 \\
\hline TOTAL & 17 & 21 & 26 & 40 & 65 & 71 \\
\hline
\end{tabular}

Attention : pour la banlieue lointaine et la campagne, les chiffres concernent la surface totale des départements et non la seule partie

(Tableau établi d'après la base de données Météo-France COLCHIQUE)

16 Au-delà de la densité des postes, la représentativité géographique des sites de mesure constitue un autre point essentiel concernant la climatologie. Tous les problèmes de représentativité (et de qualité) de la mesure sont d'ailleurs au cœur d'une réflexion entreprise par les instances météorologiques internationales (OMM), comme en témoignent pour Météo-France les publications de Michel Leroy sur la classification des sites (1999 et 2002). Cette dimension de la recherche est particulièrement cruciale en climatologie d'échelle fine. Ici les impératifs sont différents de ceux de la prévision synoptique, et la normalisation du site devient alors un obstacle à la connaissance de la diversité climatique réelle des milieux géographiques (Cantat et Brunet 2001, 2003). En climatologie urbaine, l'espace normalisé engazonné et dégagé est-il représentatif de la ville? Pour comparer du comparable et préciser ainsi la diversité des climats urbains, la définition d'un site « type » urbain serait souhaitable...

\section{Principaux caractères et mécanismes de formation de l' ICU parisien}

17 Le diagramme thermo-isoplèthes de la figure 3 (lignes d'égal écart de température entre Paris et la campagne) montre clairement l'existence de l'ICU parisien et sa double fluctuation, saisonnière et journalière : le contraste urbain/rural est particulièrement 
marqué de nuit et en été (écart supérieur à $3^{\circ} \mathrm{C}$ ), alors que de jour il est atténué, particulièrement de la fin du printemps à l'automne (écart inférieur à $1^{\circ} \mathrm{C}$ ). La visualisation du cycle journalier de l'ICU explique pourquoi les études se limitent fréquemment à la comparaison des seules températures minimales (Tn) et maximales $(\mathrm{Tx})$, dans la mesure où celles-ci traduisent relativement bien la situation nocturne et diurne.

Cette méthode d'analyse fondée sur les deux valeurs extrêmes journalières a deux conséquences :

- elle minore les écarts réels possibles entre la ville et la campagne (réduction de 0,5 à $1^{\circ} \mathrm{C}$ );

- elle conduit à des conclusions parfois abusives sur le rythme journalier de l'ICU (on mentionne ainsi fréquemment une intensité maximale en fin de nuit, tout simplement parce que c'est l'heure où se produit généralement le minimum thermique sur lequel se fonde l'étude).

19 Si l'on ne s'intéresse pas précisément au cycle journalier de l'ICU, le recours aux seules Tn et Tx est donc recevable, de plus il nécessite quatre fois moins de données (2 valeurs extrêmes contre 8 valeurs tri-horaires)...

Le tableau 3 et la figure 4 présentent ainsi pour l'ensemble des sites représentatifs de la région parisienne les écarts thermiques moyens de Tn et Tx entre le centre urbain, les banlieues et l'espace rural environnant :

- pour les Tn : un dispositif concentrique apparaît distinctement, faisant ressortir le rôle essentiel de la proximité du centre de l'agglomération (cette dépendance est matérialisée par la faible dispersion des stations autour des courbes de régression température / distance de la figure $4 a: R^{2}$ annuel $\left.>0,9\right)$;

- pour les Tx : seul le centre de Paris se différencie véritablement des autres stations grâce à un environnement très minéralisé autour du parc de mesures, donc plus chaud (fig. 4b). Sinon, nous n'observons pas d'organisation spatiale majeure, avec en été un rôle dominant du type de milieu végétal encadrant la station météorologique : les espaces verts à végétation rase subissent un échauffement plus important que les parcs boisés qui imposent une certaine fraîcheur (la masse végétale fait écran au soleil direct et assure une plus forte évapotranspiration). Ainsi, à Paris, entre mai et octobre, le parc Montsouris est en moyenne légèrement plus frais que les aérodromes périphériques. 
Tab. 3 : Températures moyennes mensuelles sous abri du centre de l'agglomération vers la périphérie rurale (moyenne sur la période 1971-1980)

\begin{tabular}{|c|c|c|c|c|c|c|c|c|c|c|c|c|c|c|}
\hline $\mathrm{T}^{\circ}$ minimales & & $\mathrm{J}$ & $\mathrm{F}$ & $M$ & $A$ & $M$ & $\mathrm{Ju}$ & $\mathrm{Jl}$ & A & $S$ & 0 & $\mathrm{~N}$ & $\mathrm{D}$ & AN \\
\hline \multirow{2}{*}{ centre urbain } & P-St-Jacques & 3,0 & 3,9 & 5,3 & 7,2 & 10.9 & 13,8 & 15,9 & 15,9 & 13.1 & 9.7 & 5.9 & 4.2 & 9.1 \\
\hline & P-Montsouris (parc) & 2,3 & 3,2 & 4,6 & 6,5 & 10,2 & 13,1 & 15,3 & 15,2 & 12,3 & 8.9 & 5.1 & 3.4 & 8.3 \\
\hline \multirow{2}{*}{ banlieue proche } & Colombes & 2,1 & 3,0 & 4,1 & 5,9 & 9,7 & 12,6 & 14,7 & 14,5 & 11.8 & 8.5 & 4.8 & 3.1 & 7.9 \\
\hline & Joinville-le-Pont & 1,4 & 2,2 & 3,7 & 5,8 & 9,5 & 12,6 & 14,7 & 14,4 & 11.3 & 7.9 & 4.1 & 2.5 & 7.5 \\
\hline banlieue éloignée & Orly (aéroport) & 0.9 & 1,9 & 3,0 & 5,0 & 8,8 & 11,7 & 13,8 & 13,6 & 10.6 & 7.3 & 3.6 & 2.0 & 6.9 \\
\hline \multirow{2}{*}{ périphérie rurale } & Brétigny (aéroport) & 0,4 & 1,5 & 2,3 & 4,4 & 7,8 & 10,5 & 12,6 & 12.5 & 9.4 & 6.1 & 2.8 & 1.6 & 6.0 \\
\hline & Melun (aéroport) & 0,6 & 1,5 & 2,3 & 4,1 & 7,9 & 10,6 & 12,5 & 12.3 & 9.4 & 6.3 & 2.8 & 1.6 & 6.0 \\
\hline $\mathrm{T}^{\circ}$ maximales & & $\mathrm{J}$ & $\mathrm{F}$ & $M$ & A & $\mathrm{M}$ & $\mathrm{Jn}$ & Jl & A & S & 0 & $\mathrm{~N}$ & $\mathrm{D}$ & AN \\
\hline \multirow{2}{*}{ centre urbain } & P-St-Jacques & 7.0 & 9.3 & 12.3 & 15.8 & 20.1 & 23.2 & 25.5 & 26.1 & 22.4 & 16.9 & 10.7 & 8.1 & 16.5 \\
\hline & P-Montsouris (parc) & 6.3 & 8.3 & 10.9 & 14.0 & 18.1 & 21.1 & 23.4 & 23.6 & 20.3 & 15.3 & 9.8 & 7.5 & 14.9 \\
\hline \multirow{2}{*}{ banlieue proche } & Colombes & 7.1 & 8.7 & 11.1 & 14.4 & 18.7 & 21.5 & 23.8 & 24.2 & 21.0 & 15.7 & 10.5 & 8.2 & 15.4 \\
\hline & Joinville-le-Pont & 6.7 & 8.5 & 11.3 & 14.7 & 19.1 & 22.2 & 24.5 & 24.8 & 21.2 & 15.7 & 10.1 & 7.8 & 15.6 \\
\hline banlieue éloignée & Orly (aéroport) & 5.9 & 8.0 & 10.6 & 13.9 & 18.1 & 21.2 & 23.7 & 24.0 & 20.5 & 15.3 & 9.6 & 7.0 & 14.8 \\
\hline \multirow{2}{*}{ périphérie rurale } & Brétigny (aéroport) & 5.8 & 7.9 & 10.6 & 13.8 & 17.9 & 21.2 & 23.9 & 24.2 & 20.6 & 15.3 & 9.5 & 6.9 & 14.8 \\
\hline & Melun (aéroport) & 5.8 & 8.0 & 10.8 & 14.3 & 18.4 & 21.5 & 23.9 & 24.2 & 20.7 & 15.4 & 9.5 & 6.9 & 15.0 \\
\hline
\end{tabular}

(tableau de synthèse établi d'après données Météo-France)

21 Les caractères de l'ICU sont avant tout fonction de l'absorption du rayonnement solaire par les matériaux durant la journée et de sa lente restitution sous forme de chaleur durant la nuit: conversion maximale du rayonnement solaire en chaleur sensible en raison de la réduction en ville des phénomènes évapotranspiratoires rafraîchissants et stockage d'énergie dans les matériaux de construction (pour plus de développements, voir Dettwiller, 1970 et Escourrou, 1991).

Fig. 4 : Températures moyennes en fonction de la distance au centre de Paris (températures réduites au niveau de la mer de 27 stations, moyenne sur la période 1971-1980)

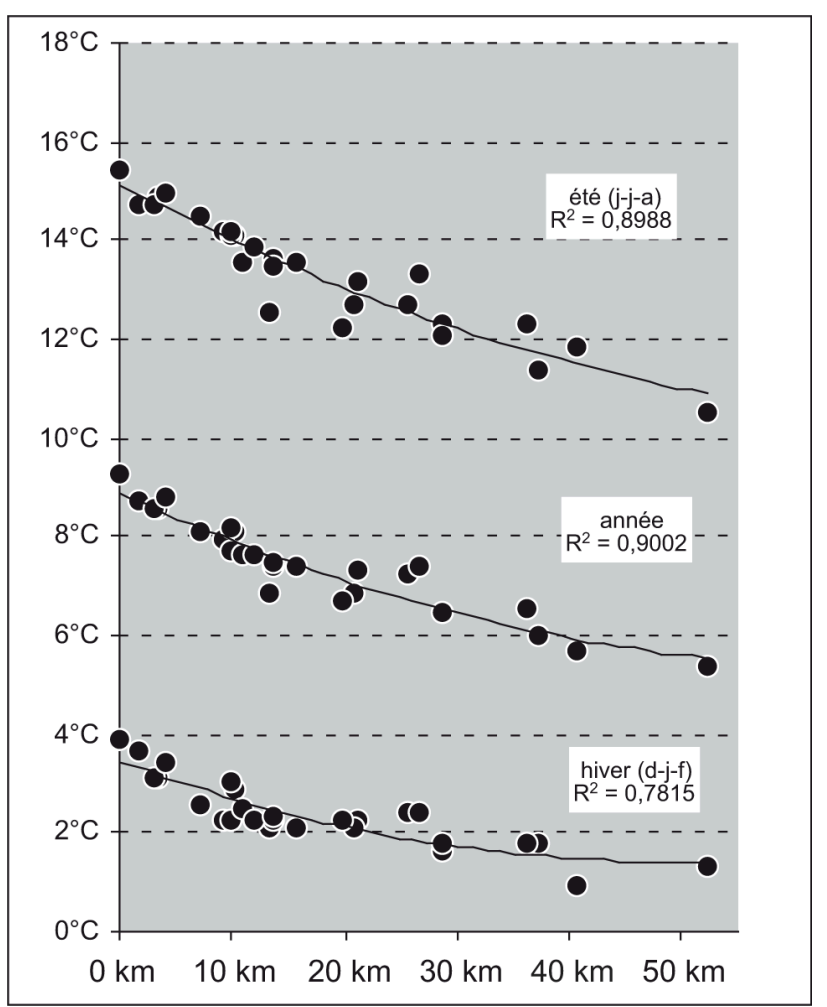

a) Température minimale 


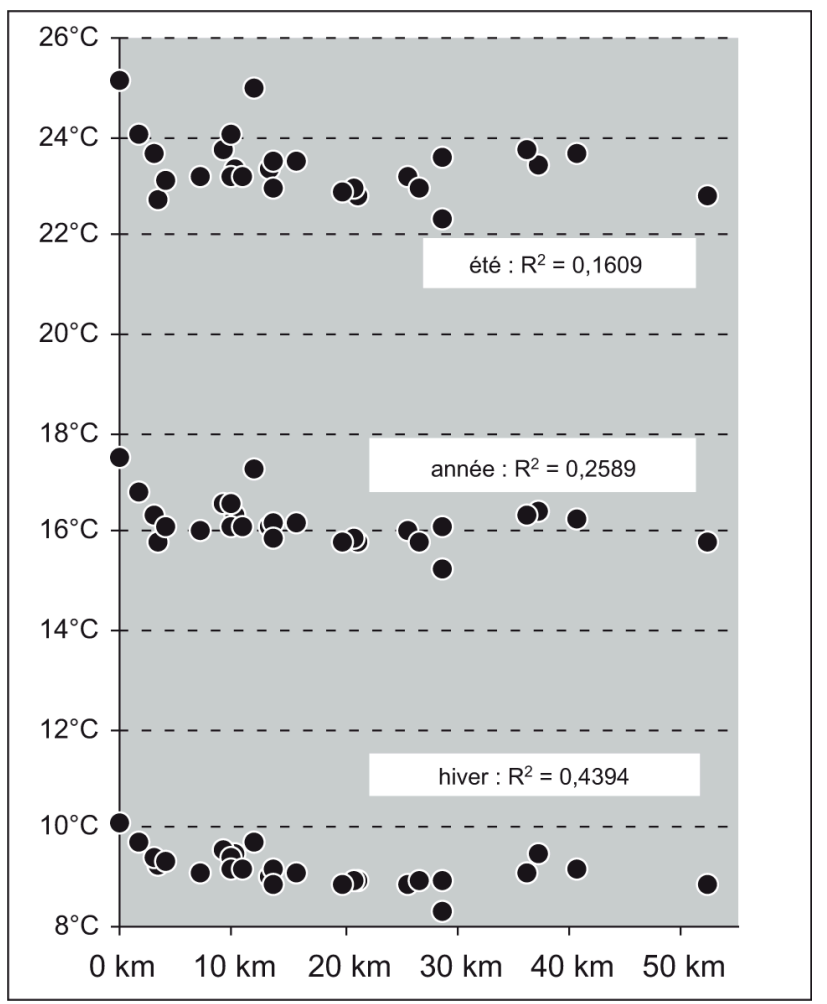

b) Température maximale

\section{L'influence des saisons sur l'intensité de l'ICU parisien}

22 Au-delà des moyennes, la succession des 360 décades de la décennie 1990-1999 démontre la grande variabilité d'intensité de l'ICU nocturne (fig. 5). Si l'écart moyen est de $3,3^{\circ} \mathrm{C}$, celui-ci oscille entre 1 et $6^{\circ} \mathrm{C}$. Cet étalement des valeurs paraît être en relation avec les saisons (fig. 6). 
Fig. 5 : Distribution fréquentielle décadaire de l'intensité de l'ICU nocturne entre 1990 et 1999 (comparaison des Tn de Paris-St-Jacques et Melun)

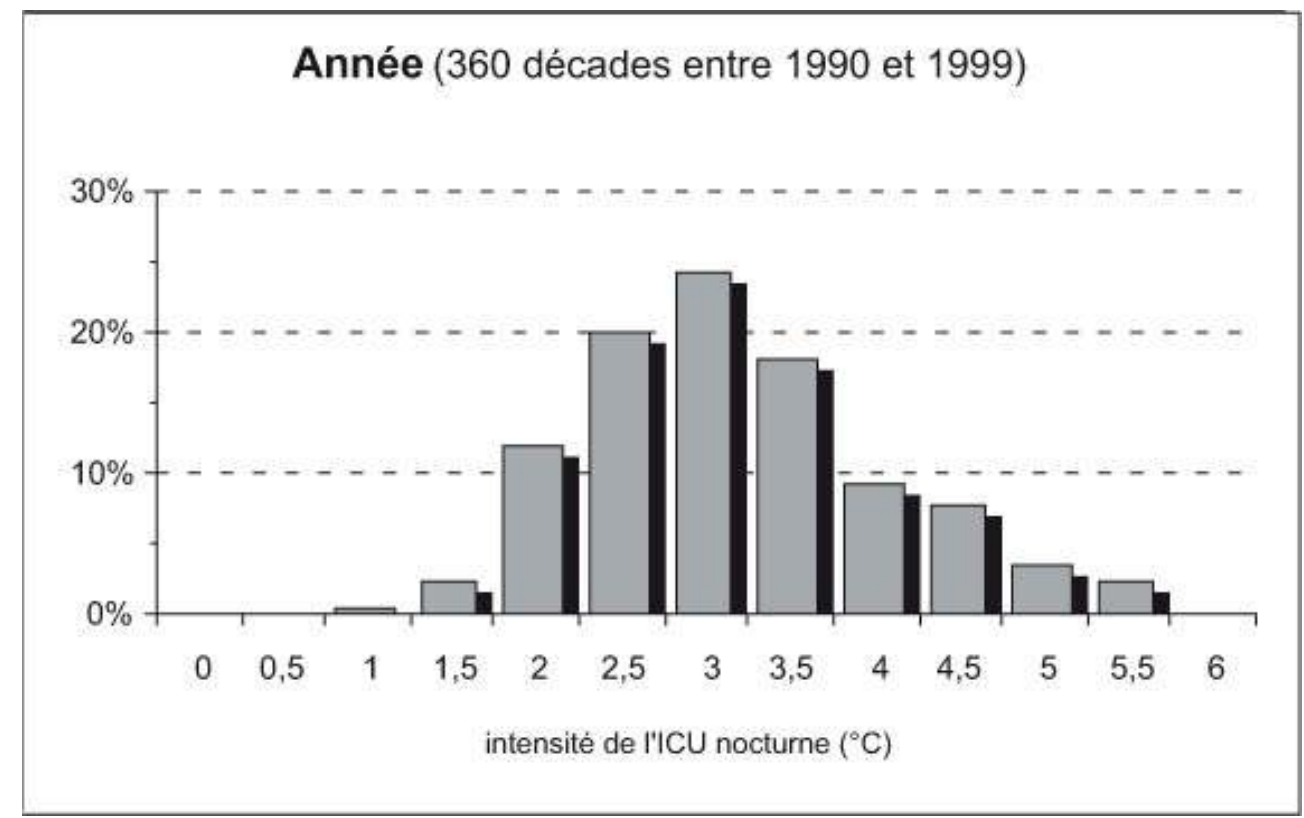

Pour les 50 décades aux ICU les plus forts (fig. 6a), l'été présente 34\% des cas, contre 6\% pour l'hiver. Inversement, sur les 50 décades aux ICU les plus faibles, l'hiver regroupe $56 \%$ des situations contre $8 \%$ en été (fig. 6b). Au pas de temps mensuel, la distinction hiver / été est encore plus nette ( $22 \%$ en août et $0 \%$ en décembre et janvier) ; elle permet aussi de constater un décalage des influences estivales génératrices potentielles de forts ICU sur le début de l'automne météorologique : $18 \%$ en septembre contre $8 \%$ en juillet seulement (étude en cours).

Fig. 6 : Répartition saisonnière des occurrences de forts et faibles ICU nocturnes entre 1990 et 1999

(calcul à partir des 50 décades extrêmes des écarts de Tn entre Paris et Melun)
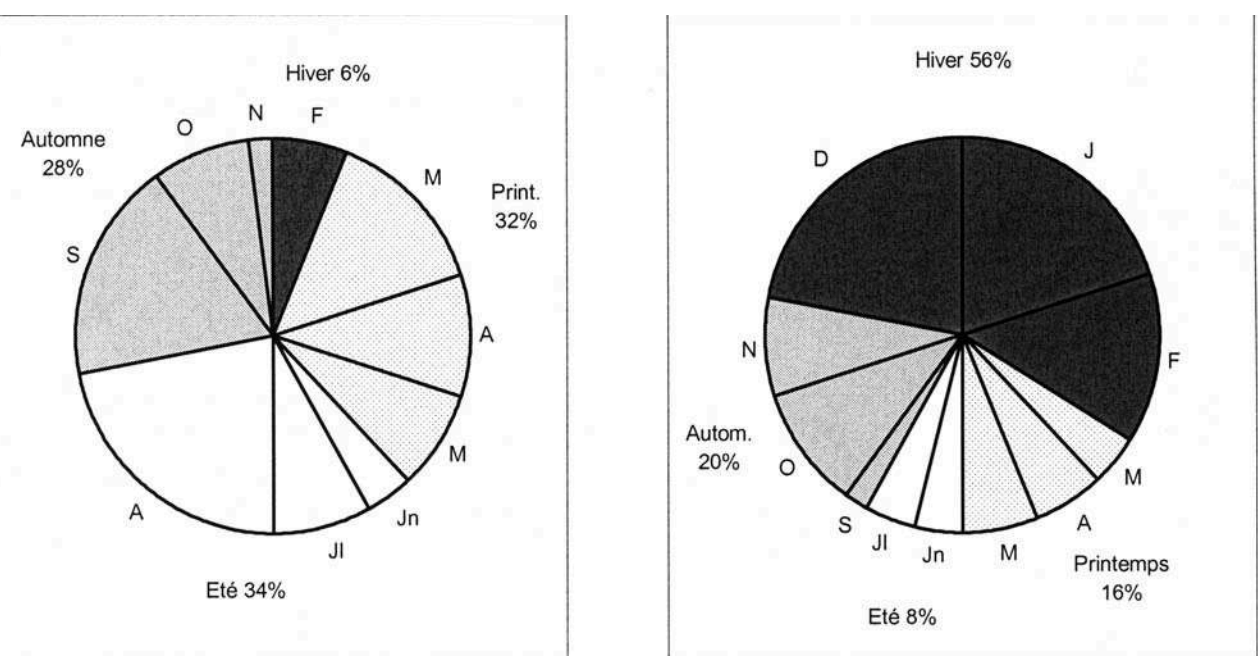

En réduisant le pas de temps à la journée, une très forte variabilité se surimpose à l'influence saisonnière (fig. 7) : si les plus forts écarts apparaissent pendant la fin de la saison chaude (août et septembre), la saison n'explique pas tout puisque certaines journées estivales présentent un très faible ICU $\left(<2^{\circ} \mathrm{C}\right)$ et inversement des «pics » (> 
$5^{\circ} \mathrm{C}$ ) peuvent apparaître au cours de l'hiver. Cette particularité du rythme de l'ICU est à mettre en relation avec la diversité des types de temps qui se succèdent à nos latitudes. Les saisons interviendraient donc avant tout comme un révélateur de la plus ou moins grande fréquence des types de temps favorables ou non à la formation de l'ICU.

Fig. 7 : Variabilité journalière de l'intensité de l'ICU nocturne parisien en 1999

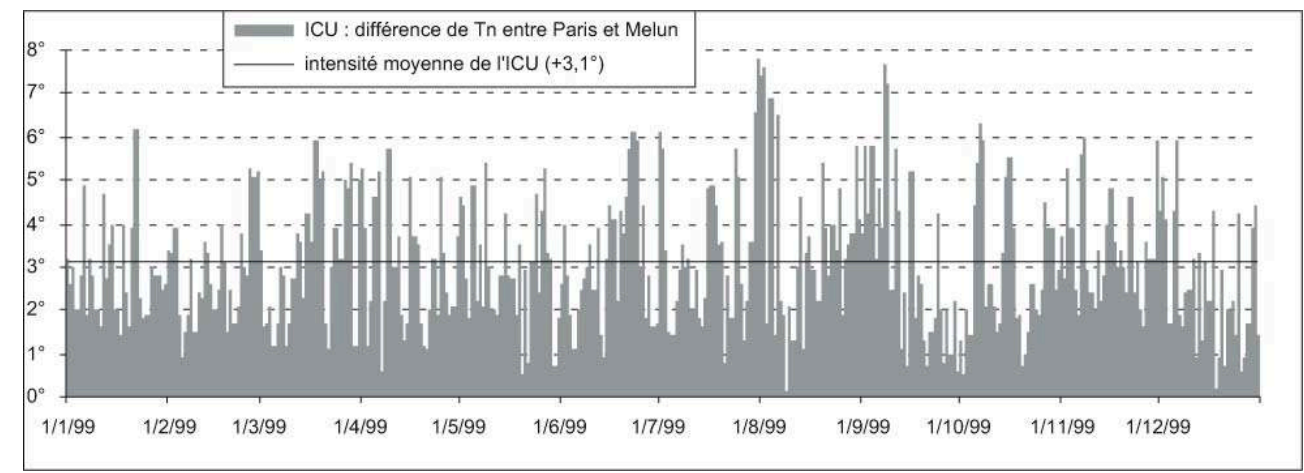

\section{L'influence des types de temps sur lîlot de chaleur parisien}

Pour confirmer l'influence des types de temps sur l'intensité de l'ICU parisien, l'étude en continu de la période du 8 au 17 janvier 1997 est particulièrement significative (fig. 8). Début janvier, une masse d'air très froid, accompagnée d'une bise perçante et d'un ciel couvert, envahit le nord de la France : l'ICU est alors pratiquement absent (oscillations entre 0 et $2^{\circ} \mathrm{C}$, sans rythme diurne particulier). Le 12 janvier, un changement de type de temps se produit : le vent faiblit et le ciel se dégage totalement. À partir du 13 et jusqu'au 17 janvier, bien qu'en plein hiver, les conditions sont alors favorables à la formation d'un ICU très marqué durant la nuit, et plus particulièrement en début de soirée et en fin de nuit $\left(>5^{\circ} \mathrm{C}\right)$. Inversement, en milieu de journée, l'espace rural se réchauffe plus rapidement sous l'action du soleil en raison de sa plus faible inertie thermique: la température de l'air sous abri «s'aligne » alors avec celle du centre de l'agglomération. Le rôle de la nébulosité et de la vitesse du vent paraît donc essentiel dans la compréhension des variations intra et inter-journalières de l'intensité de l'ICU parisien. 
Fig.8 : L'influence des types de temps sur les variations intra et inter-journalières de l'ICU (écarts des températures horaires entre Paris-St-Jacques et Melun du 8 au 17 janvier 1997)

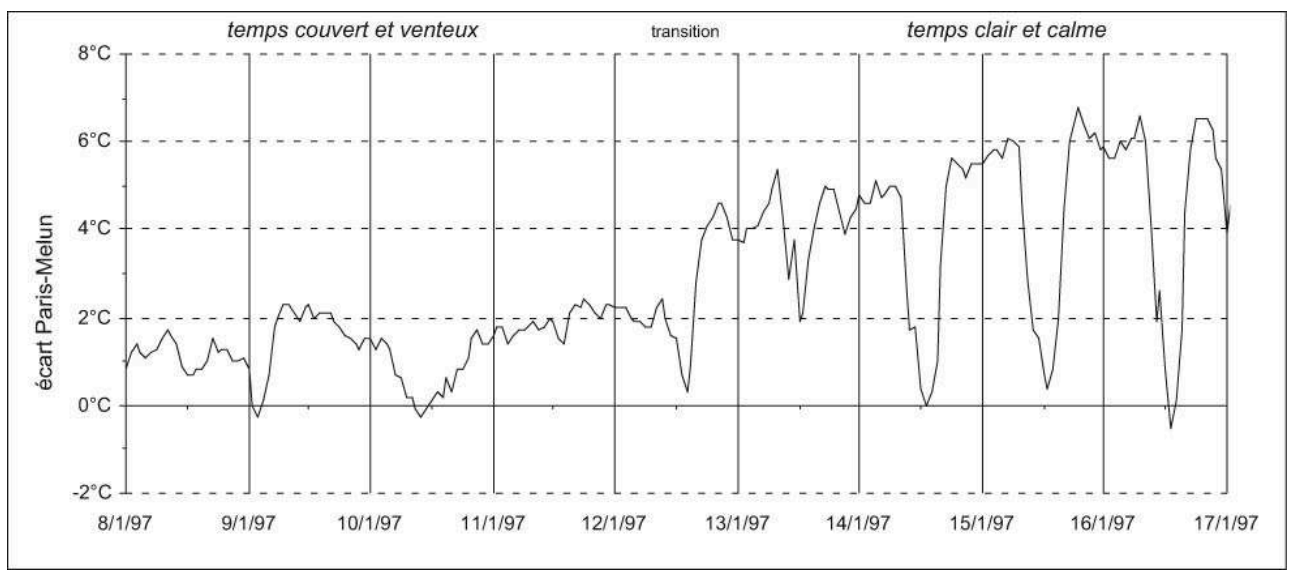

\section{Mise en évidence des paramètres discriminants dans la formation de l'ICU}

Une approche de climatologie statistique permet la mise en évidence de l'influence respective des différentes composantes atmosphériques sur la formation de l'ICU. L'association de ces composantes permet alors d'identifier un type de temps préférentiel et de proposer une approche de climatologie dynamique (cf. $5^{\mathrm{e}}$ partie).

Le traitement de l'information climatique à l'aide de techniques d'analyse multivariée confirme que la fraction d'insolation et la vitesse du vent sont les variables météorologiques les plus discriminantes pour expliquer le différentiel de température nocturne entre le centre de l'agglomération et la campagne environnante. Au pas de temps journalier, la concomitance d'un ciel clair et d'un vent faible entraîne des écarts parfois supérieurs à $10^{\circ} \mathrm{C}$ la nuit; inversement, l'ICU peut disparaître presque totalement par ciel couvert et vent fort (comme nous l'avions vu sur la figure 1).

Plus généralement, tous les facteurs propices à la clarté du ciel et à la stagnation des masses d'air, d'origines topographiques (abri) et/ou atmosphériques, semblent donc favorables au développement de l'ICU. La plus grande fréquence des types de temps clair et calme en été explique pourquoi, paradoxalement, l'ICU parisien est moins prononcé en hiver $\left(2,5\right.$ contre $\left.3,4^{\circ} \mathrm{C}\right)$ malgré l'apport de chaleur lié au chauffage. En effet, dans les régions de climat tempéré océanique, l'hiver est en moyenne une saison plus nébuleuse ( 23 contre $45 \%$ de fraction d'insolation) et plus ventée ( 4,1 contre $3,3 \mathrm{~m} /$ s) que l'été. Comme le soulignait J. Dettwiller « la ventilation intense, durant la saison froide, élimine une grande partie de cette chaleur artificielle, tandis que durant l'été, où les vents restent généralement faibles, l'influence de la nature du sol devient prépondérante » (Dettwiller, 1970).

Toutes saisons confondues, en considérant maintenant les 20 décades aux ICU les plus marqués durant la décennie 1990-1999, la figure 9 traduit graphiquement l'importance du vent et de la nébulosité. La pression atmosphérique apporte une information redondante dans la mesure où les hautes pressions sont synonymes fréquemment de temps calmes et clairs et les basses pressions de temps agités et couverts. Les exceptions à la règle expliquent alors des ICU «atypiques", comme nous le verrons dans la cinquième partie. De plus, la pression au sol présente une variation saisonnière 
liée au niveau de température, ce qui limite le caractère discriminant de son intensité : en hiver, les anticyclones «thermiques » développés en air froid sont puissants (valeurs oscillant entre 1030 et $1040 \mathrm{hPa}$ assez fréquentes), alors qu'en été les hautes pressions sont plus souvent « dynamiques » et moins marquées au sol en raison des températures plus élevées (de 1020 à 1025 hPa généralement).

Fig. 9 : Relation entre l'intensité de l'ICU nocturne et divers paramètres météorologiques (moyenne des 20 décades extrêmes sur la décennie 1990-1999)

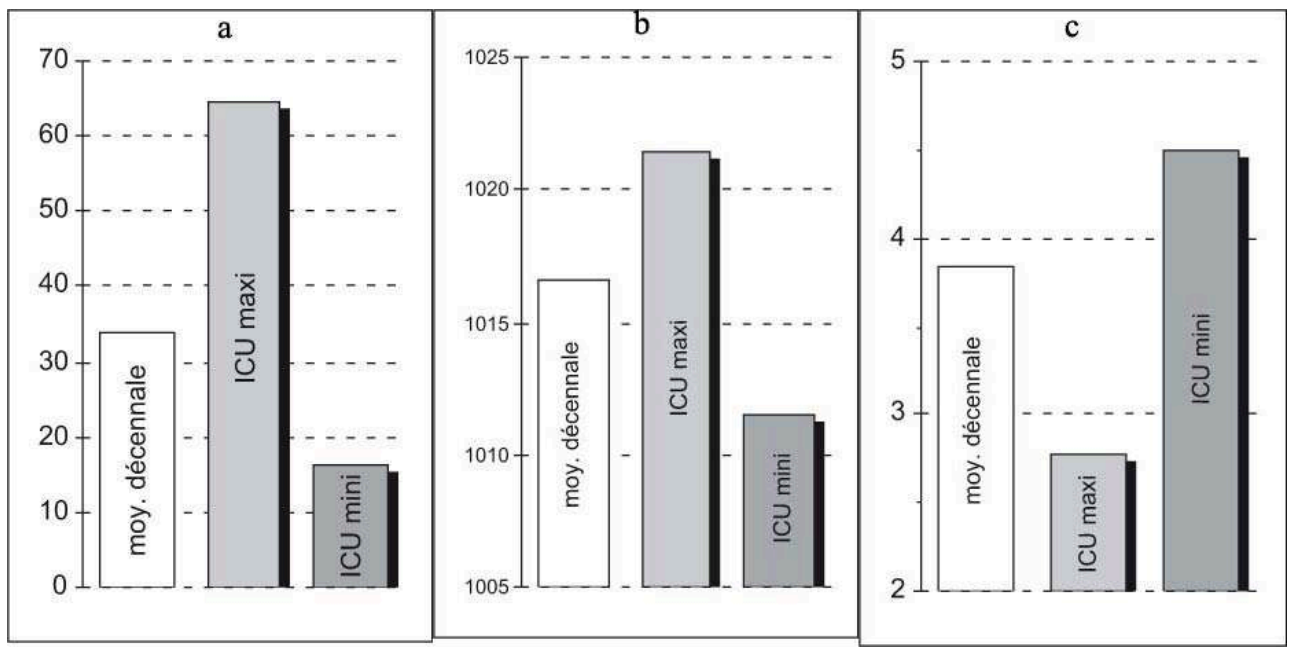

9a: fraction d'isolation (\%)

$9 \mathrm{~b}$ : pression atmosphérique $(\mathrm{hPa})$

$9 \mathrm{c}$ : vitesse moyenne du vent $(\mathrm{m} / \mathrm{s})$

\section{Approche de synthèse des types de temps favorables à l'ICU}

Au pas de temps mensuel, les seuils de $50 \%$ et de $3 \mathrm{~m} / \mathrm{s}$ ont été choisis pour qualifier une forte fraction d'insolation et un vent faible. Pris séparément, ce ne sont pas des conditions rares en région parisienne (respectivement 20,0 et $15,8 \%$ ), mais associées elles ne représentent plus que $6,7 \%$ des cas (fig. 10) et correspondent à des ICU supérieurs à $4,2^{\circ} \mathrm{C}$.

Fig. 10 : Fréquence annuelle des types de temps favorables à un fort ICU (à partir des 120 mois de la période 1990-1999)
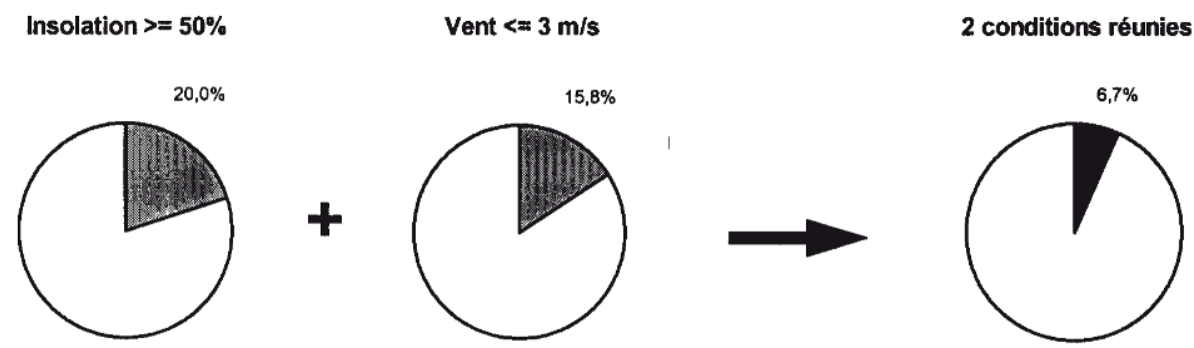

31 Le même calcul effectué au pas de temps décadaire offre plus de cas en raison d'une «empreinte» des types de temps plus facilement exprimée à ce pas de temps plus 
court : les fortes fractions d'insolation s'élèvent alors à $24,4 \%$, les vents faibles à $22,8 \%$ et les deux conditions réunies à 10,8\% (avec un ICU moyen de 4,6 $6^{\circ} \mathrm{C}$ ).

En divisant en quatre classes le vent et la fraction d'insolation, le calendrier par décade du tableau 4 fait bien ressortir le rôle essentiel des saisons sur l'occurrence des types de temps plus ou moins favorables à l'ICU (18,9\% de très favorable en été contre $2,2 \%$ en hiver), et leur dépendance majeure avec le «balancement» moyen des centres d'action :

- une période très favorable en juillet-août, c'est-à-dire quand l'anticyclone subtropical remonte aux latitudes moyennes et apporte sa subsidence. Cette période débute quelquefois dès le printemps et se prolonge parfois jusqu'au début de l'automne (le mois de septembre est très fréquemment favorable) ;

- inversement, de novembre à janvier, des conditions généralement très défavorables en raison de l'activité dominante des perturbations liée aux influences subpolaires océaniques.

Ces observations confirment les hypothèses pressenties en analysant la distribution saisonnière de l'ICU (fig. 6).

Fig. 11 : Rôle de la vitesse du vent et de la fraction de ciel clair sur l'intensité de l'ICU nocturne parisien (représentation graphique en thermo-isoplèthes à partir des 360 décades de la période 1990-1999)

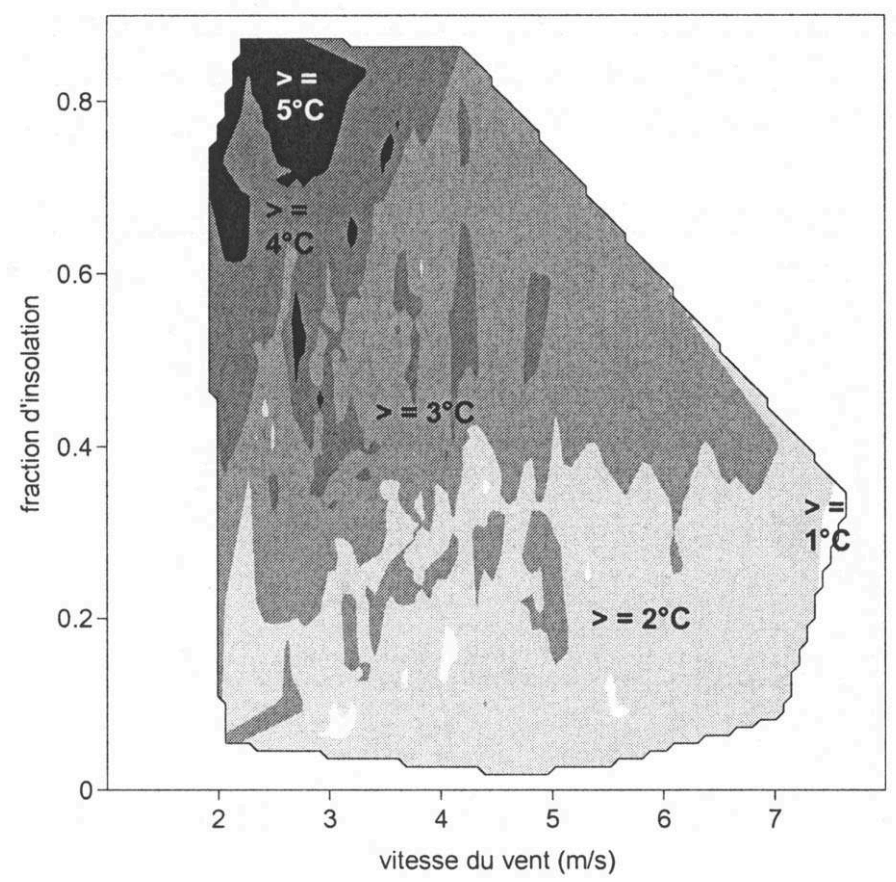

Cette figure traduit la double dépendance majeure de l'ICU : la « poche » des ICU > = $5^{\circ} \mathrm{C}$ englobe approximativement les deux conditions requises précédemment $(50 \%$ d'insolation et moins de $3 \mathrm{~m} / \mathrm{s}$ de vent moyen). Inversement, par vent supérieur à $7 \mathrm{~m} /$ s, l'ICU tend à disparaître. Au-delà de cette allure globale bien définie, le tracé irrégulier des isoplèthes traduit l'intervention d'autres paramètres météorologiques non pris en compte dans ce traitement graphique mais susceptibles d'apporter des nuances. 
Tab. 4 : Calendrier des types de temps favorables à l'ICU entre 1990 et 1999

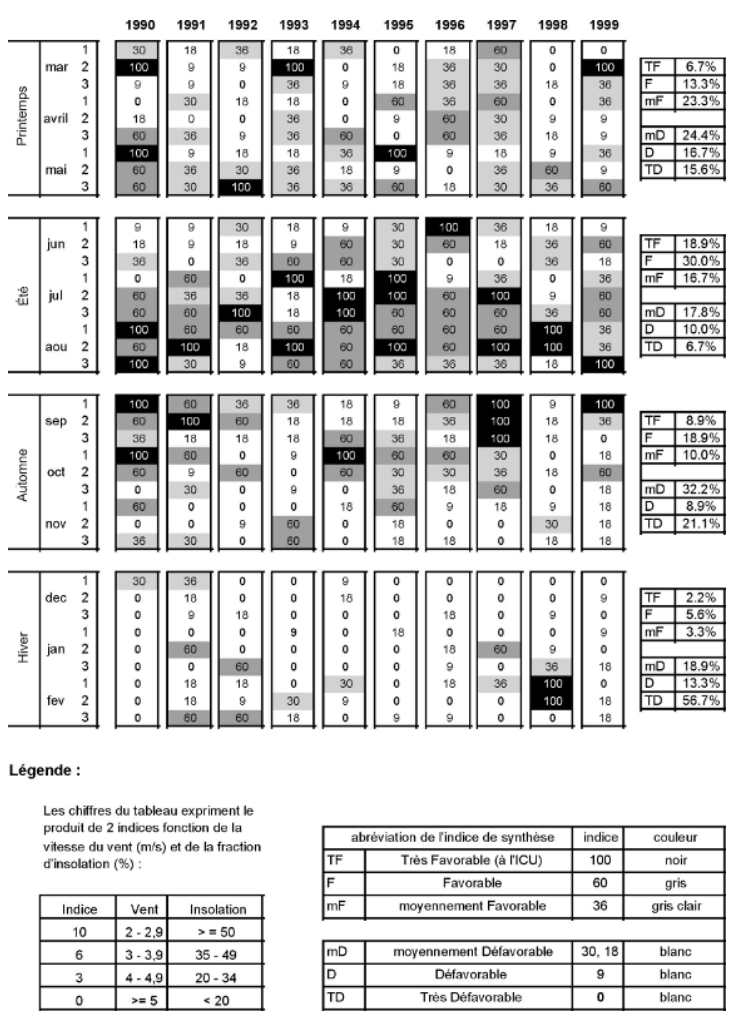

La direction du vent permet de préciser davantage les types de temps favorables à l'ICU (fig. 12). Sur les 1094 jours de l'analyse, si l'on considère par exemple les 50 jours « extrêmes » (environ 5\% des ICU maximums et minimums), deux types de temps très opposés se dégagent clairement (fig. 12c) :

- faibles ICU (moyenne : + 0,5 $\left.{ }^{\circ} \mathrm{C}\right)$ : le vent est à dominante sud-ouest, sa vitesse élevée $(5,1 \mathrm{~m} / \mathrm{s})$ et les calmes sont totalement absents $(0 \%)$; le ciel est très couvert (fraction d'insolation de $17,5 \%)$ et la pression atmosphérique généralement basse $(1009,9 \mathrm{hPa})$;

- forts ICU (moyenne : + 7,3ㄷ) : le vent est à dominante nord-est, sa vitesse est faible $(1,2 \mathrm{~m} / \mathrm{s})$ et les calmes sont très fréquents $(20 \%)$; le ciel est généralement bien dégagé (fraction d'insolation de 65\%) et la pression atmosphérique généralement élevée (1022,6 hPa).

En prenant les 200 jours « extrêmes » (fig. 12b), les valeurs apparaissent un peu moins tranchées entre les conditions enregistrées lors des faibles et des forts ICU, mais les conclusions sont identiques: la comparaison entre la rose des vents "normale " (fig. 12a) et celles établies dans les cas de forts ICU montre la dominance des flux d'est à nord-est (fig. $12 \mathrm{~b}$ et $12 \mathrm{c}$ ). Ces directions privilégiées, peu fréquentes en "moyenne ", sont en accord avec les situations synoptiques propices aux types de temps clair et calme générateurs de forts ICU, généralement dues à des anticyclones dirigeant vers la France des masses d'air continental sec et peu turbulent. Il est rare que ce type de temps persiste sur une décade entière, et a fortiori sur un mois, ce qui explique la fréquence relativement faible des très forts contrastes thermiques " moyens " entre la ville et la campagne sous notre climat dominé par des flux d'ouest à sud-ouest soutenus et porteurs de nuages en raison de leur provenance océanique. Dans sa thèse sur le climat du bassin Parisien, Pierre Pédelaborde (1958) précise que la «durée d'une période homogène est de trois jours » en moyenne. 
Fig. 12 : Relation entre direction du vent et intensité de l'ICU parisien en fin de nuit
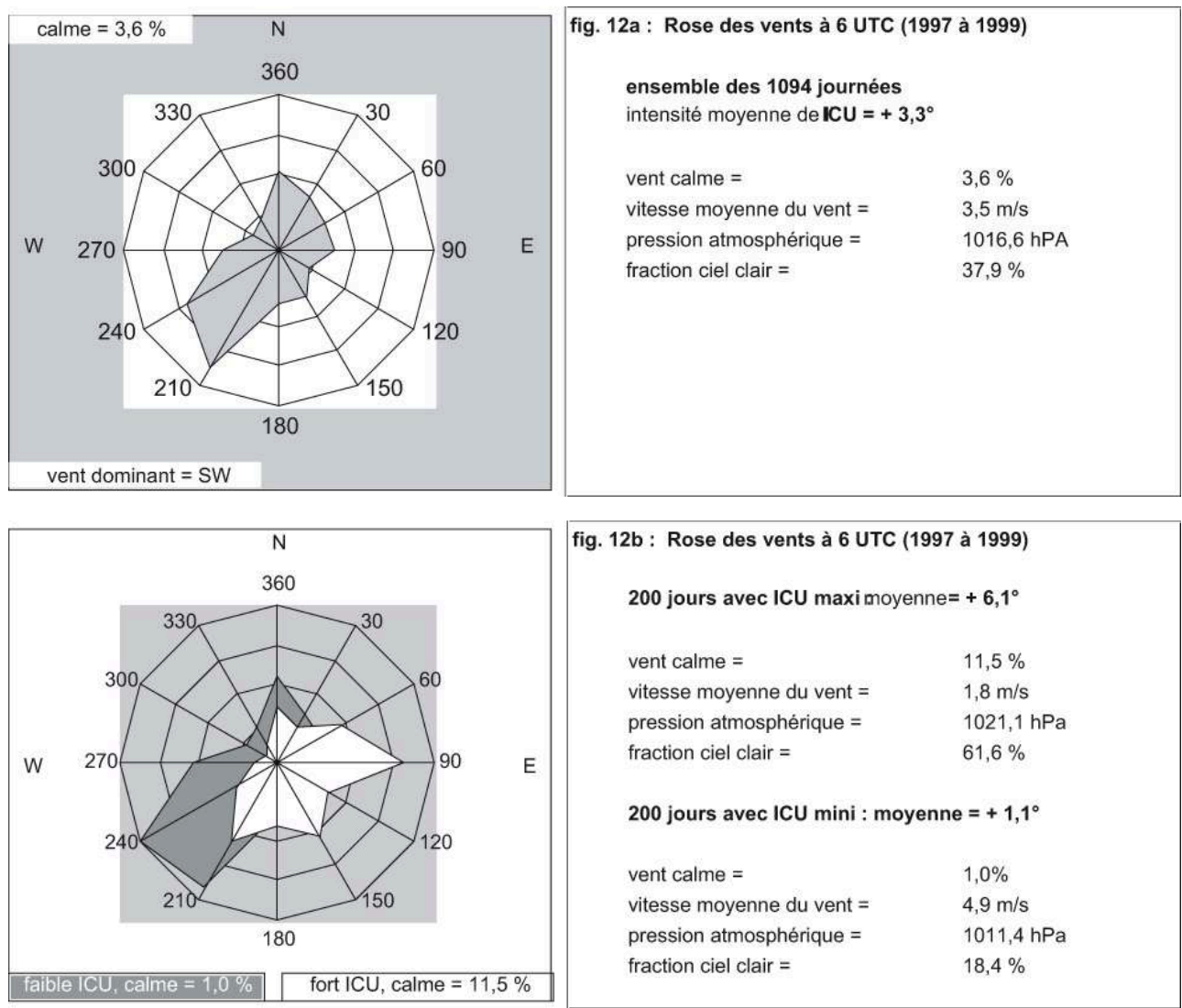

fig. 12b : Rose des vents à 6 UTC (1997 à 1999)

200 jours avec ICU maxi moyenne $=+6,1^{\circ}$

$\begin{array}{ll}\text { vent calme }= & 11,5 \% \\ \text { vitesse } \text { moyenne du vent }= & 1,8 \mathrm{~m} / \mathrm{s} \\ \text { pression atmosphérique }= & 1021,1 \mathrm{hPa} \\ \text { fraction } & 61,6 \%\end{array}$

fraction ciel clair $=$

200 jours avec ICU mini : moyenne $=+1,1^{\circ}$

vent calme $=$

$1,0 \%$

vitesse moyenne du vent $=\quad 4,9 \mathrm{~m} / \mathrm{s}$

pression atmosphérique $=\quad 1011,4 \mathrm{hPa}$

fraction ciel clair $=\quad 18,4 \%$

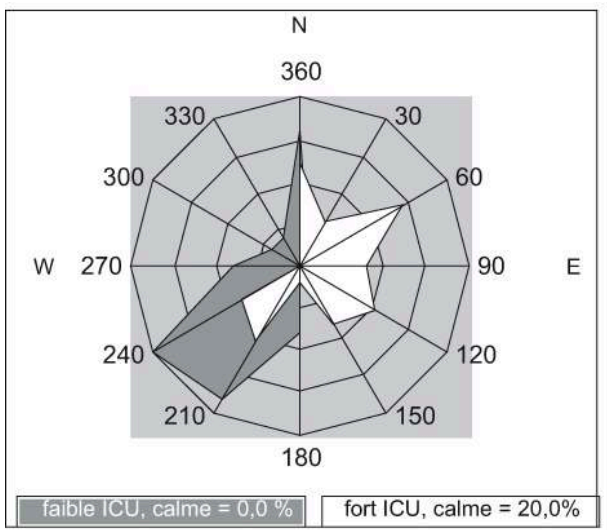

fig. 12c : Rose des vents à 6 UTC (1997 à 1999)

50 jours avec ICU maxi : moyenne $=+7$,

$\begin{array}{ll}\text { vent calme }= & 20,0 \% \\ \text { vitesse } \text { moyenne du vent }= & 1,2 \mathrm{~m} / \mathrm{s} \\ \text { pression atmosphérique }= & 1022,6 \mathrm{hPa} \\ \text { fraction ciel clair }= & 65,0 \%\end{array}$

50 jours avec ICU mini : moyenne $=+0,5^{\circ}$

vent calme $=\quad 0,0 \%$

vitesse moyenne du vent $=\quad 5,1 \mathrm{~m} / \mathrm{s}$

pression atmosphérique $=\quad 1009,9 \mathrm{hPa}$

fraction ciel clair $=\quad 17,5 \%$

\section{Modélisation de l'intensité de l'ICU parisien}

À partir des données mensuelles sur la période 1990-1999, il a été possible de «prédire » l'intensité de l'ICU nocturne parisien en fonction de la fraction de ciel clair (exprimée ici par le pourcentage d'insolation: Insol) et de la vitesse moyenne du vent $(\mathrm{Vm}$, en $\mathrm{m} / \mathrm{s})$. La régression linéaire multiple a été volontairement limitée à ces deux paramètres car les autres régresseurs (insolation, température, pression...) n'étaient pas statistiquement significatifs $d u$ fait de leur information en grande partie redondante (faible coefficient de corrélation partielle: avec 3,4 ou 5 variables, R ne 
change pratiquement pas). La figure 13 illustre graphiquement l'application de l'équation suivante :

ICU = 0,035 Insol - 0,177 Vm + 2,782 (avec R = 0,819)

Fig. 13 : Modélisation de l'ICU nocturne parisien mensuel moyen sur la période 1990-1999

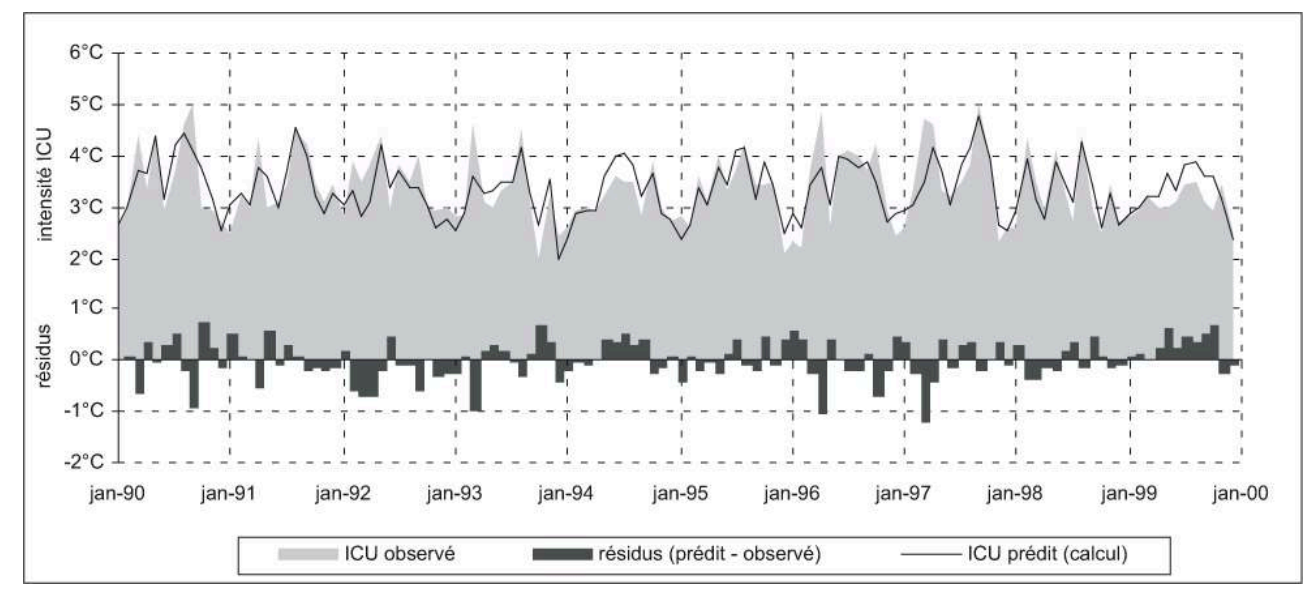

Cette modélisation constitue une simplification d'une réalité très complexe dont les mécanismes pourraient être approfondis en analysant les résidus qui montrent bien que l'ajustement par l'équation de régression est loin d'être parfait.

\section{Les « déformations » de l'îlot de chaleur parisien selon les types de temps}

L'étude des écarts de température entre Paris, ses différentes banlieues et la campagne environnante traduit la diversité des formes que prend la transition entre la «chaleur urbaine » et la "fraîcheur rurale ». Cette cartographie de l'ICU présente des physionomies très mouvantes d'un jour à l'autre selon les types de temps et la direction du vent. Une présentation synthétique des principales conclusions est proposée ici sous la forme de trois cartes de situations types.

\section{Les cas de forts ICU : présence simultanée d'un vent faible et d'un ciel clair}

La stagnation de l'air dans les basses couches et le «jeu » différencié des processus radiatifs entre la ville et la campagne conduit à la mise en place d'un ICU prononcé.

-L'exemple du 5 octobre 1997 est révélateur de ce type de situation (fig. 14). Au matin, la température au centre de Paris est déjà de $12,9^{\circ} \mathrm{C}$ alors que, sur la campagne environnante, elle descend fréquemment en-dessous de $4^{\circ} \mathrm{C}$. L'écart avec la station la plus froide atteint $11,7^{\circ} \mathrm{C}\left(1,2^{\circ} \mathrm{C}\right.$ au Châtelet-en-Brie à $53 \mathrm{~km}$ au sud-sud-est de Paris) et $8,9^{\circ} \mathrm{C}$ avec Melun, station rurale de référence $\left(4,0^{\circ} \mathrm{C}, 37 \mathrm{~km}\right.$ au sud-est de Paris). Ce très fort ICU «bénéficie » certainement d'un effet d'accumulation thermique dû à une période de temps clair et calme persistant depuis plus de deux semaines, associée à des conditions barométriques de type estival (anticyclone dynamique d'altitude sur l'Europe).

Phénomène original présent également en ce 5 octobre, par vent léger on observe 
systématiquement une élévation de la température sur plusieurs kilomètres "sous le vent » de Paris. Ici, le flux lent de sud et sud-est dominant entraîne une déformation de l'ICU en aval de Paris, matérialisée par la forme en poire de l'isotherme $8^{\circ} \mathrm{C}$ s'étirant jusqu'aux limites de l'agglomération vers le nord-ouest. Au nord de Paris, l'isotherme $6^{\circ}$ déborde largement sur les espaces agricoles de la Plaine de France, alors qu'au sud il se rapproche de la proche banlieue, traduisant ainsi une avancée de la "fraîcheur rurale » au-dessus de l'agglomération en amont de la capitale.

Le bilan thermique très variable des banlieues s'explique donc à la fois par le rôle des facteurs géographiques locaux (site et type d'urbanisation) mais aussi par l'exportation de chaleur en provenance du centre de l'agglomération et de fraîcheur en provenance de la campagne.

Fig. 14 : Cartographie de l'ICU nocturne par temps clair et peu venté (secteur sud à sud-est) Fort ICU : valeur maximale de $11,7^{\circ} \mathrm{C}$ (valeur moyenne $=6,7^{\circ} \mathrm{C}$ )

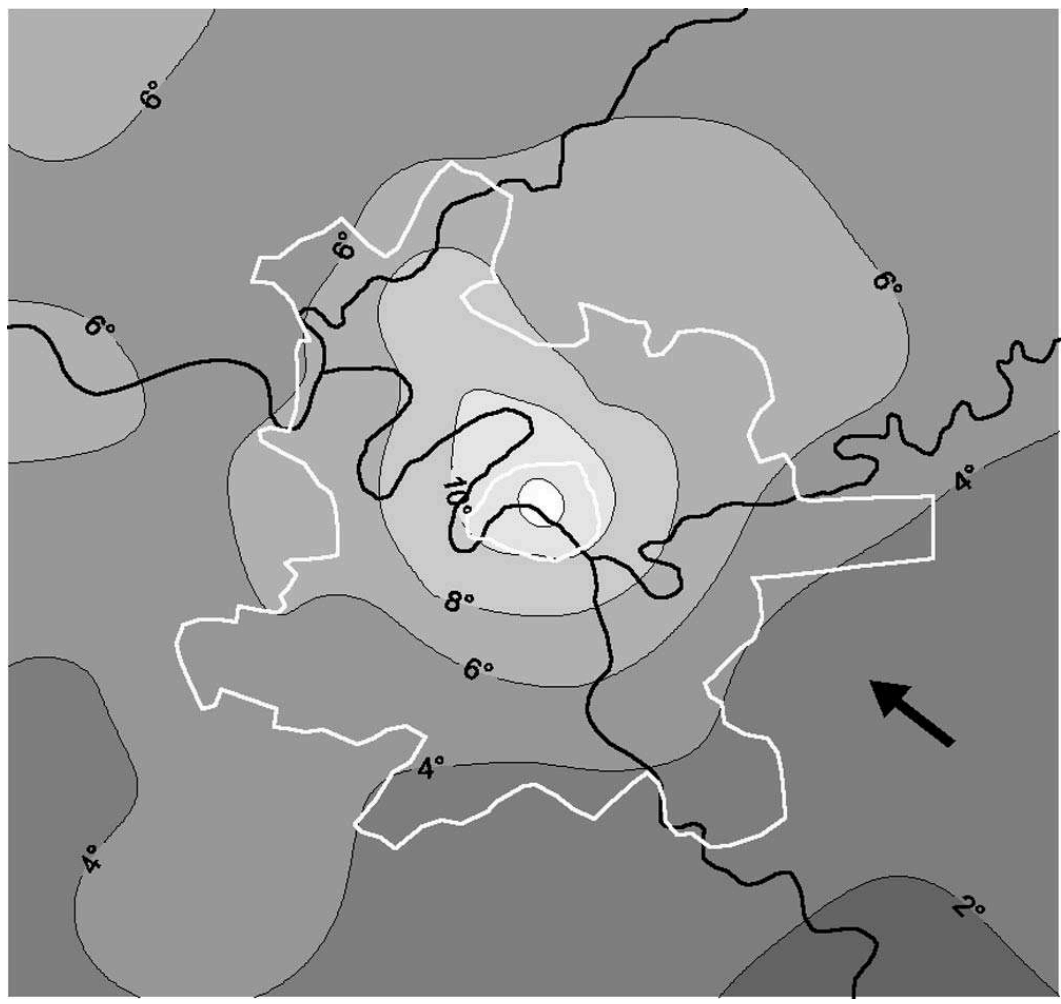

ICU du 5 octobre 1997

Contours de Paris et de l'agglomération en blanc ; cours d'eau en noir

- Parfois, en l'absence de vent du gradient, la « forte différence de température entre le centre et la périphérie engendre des vents locaux assez semblables aux brises de mer que les anglo-saxons ont baptisés brises de campagnes. (...) Dans ce cas le vent se déplace des parties les plus froides vers les parties les plus chaudes de l'agglomération. Ces brises peuvent être assez épaisses et se faire sentir sur une part importante de l'Ilede-France » (Escourrou, 1986). Les « brises de campagne " généralisées occasionnent un ICU spatialement restreint mais intense (fig. 15). Dès le coucher du soleil, le temps clair et calme engendre une couche d'inversion thermique relativement épaisse sur la campagne, mais pas sur Paris en raison de l'énergie emmagasinée dans le substratum restituée aux basses couches de l'atmosphère.

Tel fut le cas le 10 mars 1997. A minuit, le radiosondage de Trappes (27 km à l'ouest de 
Paris sur le Plateau de Beauce) montrait déjà une inversion de température supérieure à $5^{\circ} \mathrm{C}$ sur moins de 400 mètres d'épaisseur : 5,4 ${ }^{\circ} \mathrm{C}$ à $168 \mathrm{~m}$ d'altitude (sol), contre $10,6^{\circ} \mathrm{C}$ à $508 \mathrm{~m}$. A Paris, la température minimale fut de $8,8^{\circ} \mathrm{C}$, alors qu'aux limites de l'agglomération des petites gelées sous abri étaient observées (entre 0 et $-1^{\circ} \mathrm{C}$ à $\mathrm{Creil}$ au nord, Condécourt au nord-ouest, Achères à l'ouest et à seulement $22 \mathrm{~km}$ de Paris, StLéger-en-Yvelines au sud-ouest, Brétigny au sud ou encore au Châtelet-en-Brie au sudest).

Fig. 15 : Cartographie de l'ICU nocturne par temps clair et convergence des vents (brise de campagne)

Fort ICU : valeur maximale de $9,4^{\circ} \mathrm{C}$ (valeur moyenne $=7,0^{\circ} \mathrm{C}$ )

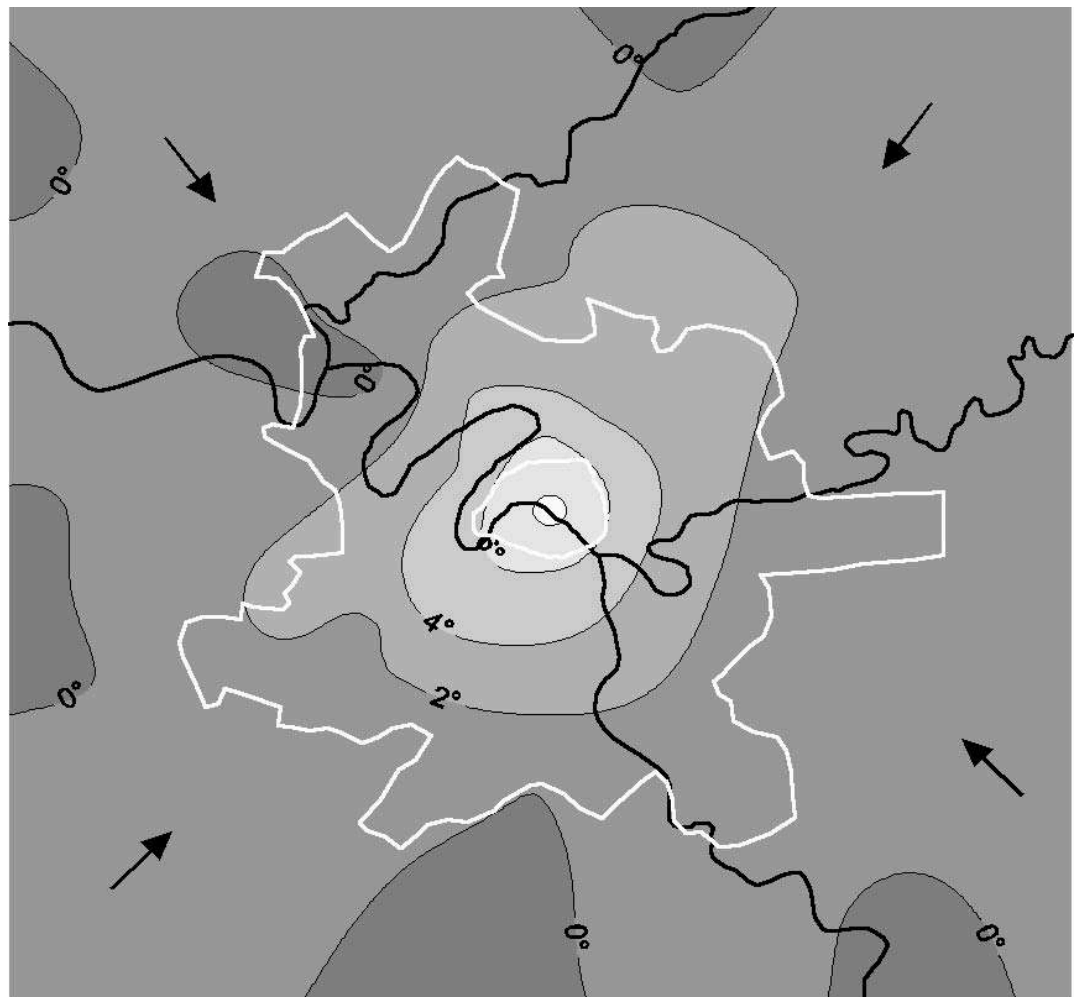

ICU du 10 mars 1997

Contours de Paris et de l'agglomération en blanc ; cours d'eau en noir

Plus généralement, en 1997, à 6 heures UTC, des vents faibles de directions opposées ont été observés à 12 reprises (3\%) entre la banlieue nord (aéroport du Bourget, $13 \mathrm{~km}$ ) et la banlieue sud (aéroport d'Orly, $15 \mathrm{~km}$ ) de Paris :

- sept cas correspondaient à des brises de campagne généralisées (comme le 10 mars 1997), avec comme facteur déclenchant un fort ICU $\left(7,2^{\circ} \mathrm{C}\right.$ en moyenne) provoqué par l'association d'un ciel clair et de vents calmes. Une importante inversion thermique était systématiquement observée dans les basses couches (voir exemple pour 4 dates dans le tableau 5);

- plus curieusement, en novembre-décembre, trois cas de brise se sont produits malgré la présence d'un faible ICU $\left(1,4^{\circ} \mathrm{C}\right.$ en moyenne) dû à un ciel totalement couvert depuis plusieurs jours. Dans ces conditions originales, peut-être s'agissait-il alors d'une convergence provoquée par la seule différence de température ville/campagne induite par les rejets de chaleur anthropiques en l'absence totale de vent synoptique ? Le faible ICU traduirait alors la contribution thermique de l'agglomération à cette saison (activités 
industrielles, transport et chauffage). Ici, aucune inversion thermique significative dans la très basse troposphère n'a été observée ;

- les deux derniers cas paraissent dus à des vents locaux, sans réelle assimilation possible à des phénomènes de brise de campagne.

Tab. 5 :Température de l'air à différents niveaux d'altitude par «brises de campagne » (radiosondages de Trappes à 00 heure UTC en 1997)

\begin{tabular}{|l|l|c|c|c|c|c|c|}
\hline \multirow{2}{*}{ le 10 mars } & altitude & $168 \mathrm{~m}$ & $256 \mathrm{~m}$ & $508 \mathrm{~m}$ & $902 \mathrm{~m}$ & $1599 \mathrm{~m}$ & $2406 \mathrm{~m}$ \\
\cline { 2 - 9 } & température & $5,4^{\circ}$ & $6,8^{\circ}$ & $\mathbf{1 0 , 6 ^ { \circ }}$ & $8,2^{\circ}$ & $7,4^{\circ}$ & $3,0^{\circ}$ \\
\hline \multirow{2}{*}{ le 24 avril } & altitude & $168 \mathrm{~m}$ & $256 \mathrm{~m}$ & $\mathrm{x}$ & $814 \mathrm{~m}$ & $1497 \mathrm{~m}$ & $2404 \mathrm{~m}$ \\
\cline { 2 - 9 } & température & $4,6^{\circ}$ & $\mathbf{9 , 6 ^ { \circ }}$ & $\mathrm{x}$ & $5,2^{\circ}$ & $-0,5^{\circ}$ & $-3,1^{\circ}$ \\
\hline \multirow{2}{*}{ le 21 août } & altitude & $168 \mathrm{~m}$ & $220 \mathrm{~m}$ & $406 \mathrm{~m}$ & $835 \mathrm{~m}$ & $1558 \mathrm{~m}$ & $2341 \mathrm{~m}$ \\
\cline { 2 - 9 } & température & $18,8^{\circ}$ & $23,2^{\circ}$ & $\mathbf{2 4 , 0 ^ { \circ }}$ & $20,6^{\circ}$ & $15,0^{\circ}$ & $8,6^{\circ}$ \\
\hline \hline \multirow{2}{*}{ le 4 octobre } & altitude & $168 \mathrm{~m}$ & $217 \mathrm{~m}$ & $446 \mathrm{~m}$ & $845 \mathrm{~m}$ & $1540 \mathrm{~m}$ & $2522 \mathrm{~m}$ \\
\cline { 2 - 9 } & température & $10,6^{\circ}$ & $\mathbf{1 3 , 6 ^ { \circ }}$ & $12,8^{\circ}$ & $9,2^{\circ}$ & $10,0^{\circ}$ & $9,8^{\circ}$ \\
\hline
\end{tabular}

\section{Les cas de faibles ICU : présence d'un ciel couvert et/ou d'un vent soutenu}

44 La forte nébulosité du ciel bloque les processus de formation de l'ICU et l'agitation de l'air disperse presque complètement la pellicule d'air chaud due aux activités urbaines. Dans ces conditions, l'excédent thermique de Paris et de la proche banlieue sous le vent se limite entre 1 et $2^{\circ}$, parfois moins encore par grand vent et précipitations. D'après Dettwiller (1970), la vitesse limite pour l'observation de l'ICU serait de l'ordre de $11 \mathrm{~m} /$ s.

La situation du 25 février 1997 (fig. 16) fournit un exemple très commun de configuration de l'ICU parisien par types de temps perturbés d'ouest.

Au total, cette approche spatialisée de l'ICU démontre que le bilan thermique très variable des banlieues traduit à la fois le rôle des facteurs géographiques locaux (site et type d'urbanisation), des types de temps, mais aussi l'importation de chaleur en provenance du centre de l'agglomération ou de fraîcheur en provenance de la campagne voisine. 
Fig. 16 : Cartographie de l'ICU nocturne par temps nuageux et venteux Faible ICU : valeur maximale de $2,3^{\circ} \mathrm{C}$ (valeur moyenne $=1,4^{\circ} \mathrm{C}$ )

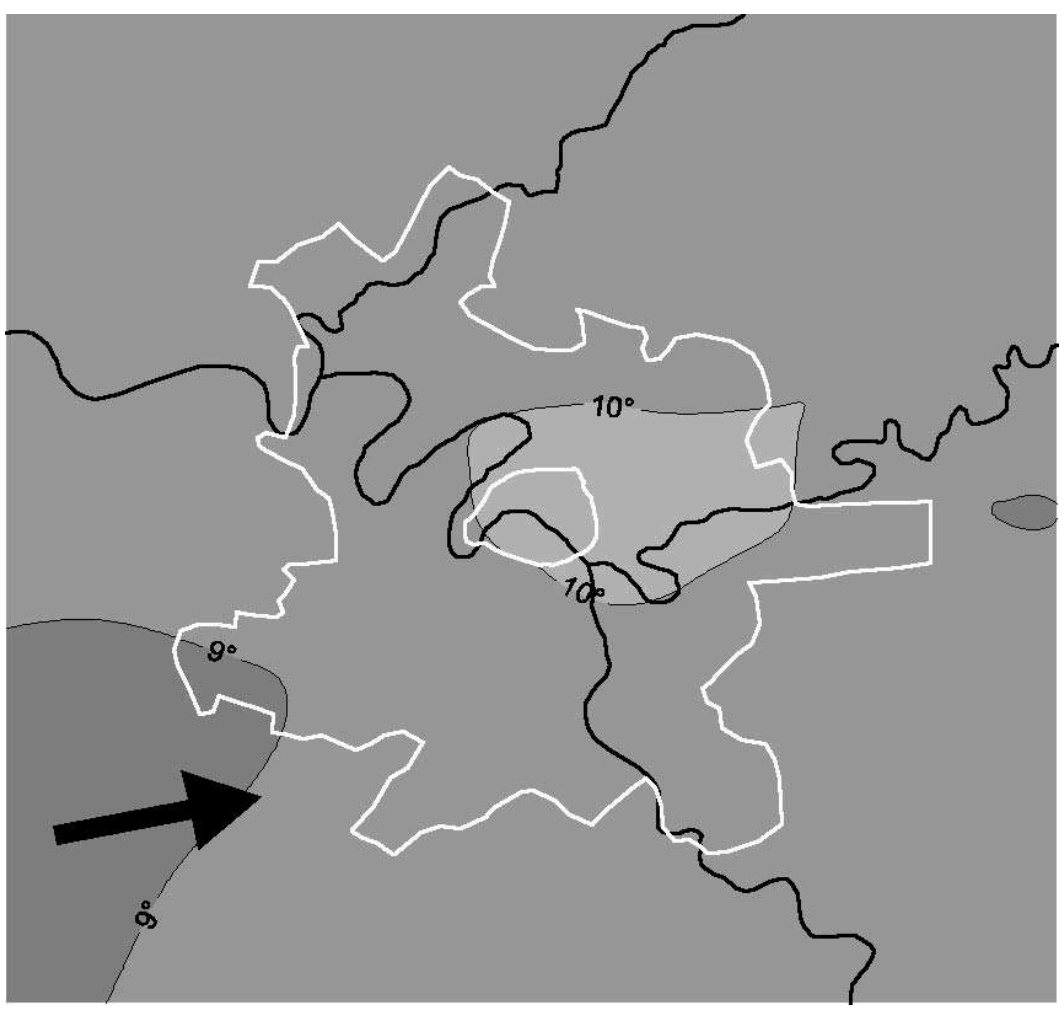

ICU du 25 février 1997

Contours de Paris et de l'agglomération en blanc ; cours d'eau en noir

\section{Les mécanismes de formation de l'îlot de chaleur urbain « éclairés " par la situation synoptique et la structure verticale de l'atmosphère}

L'étude combinée de l'ICU et de l'atmosphère dans ses trois dimensions permet d'établir un lien entre types de temps et situation synoptique. Cette analyse permet de

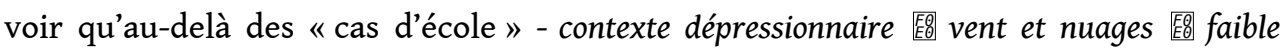

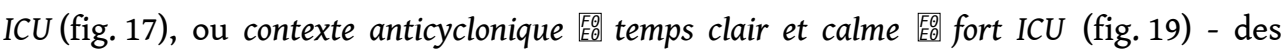
écarts de température inattendus entre la ville et la campagne peuvent aussi se produire lors de configurations barométriques a priori peu compatibles avec leur formation (fig. 18 et 20), une même situation synoptique pouvant occasionner des types de temps différents. La connaissance de la structure verticale de l'atmosphère donne alors un éclairage nouveau à ces « anomalies » dans l'intensité et la forme de l'ICU.

\section{Situation synoptique et profil vertical de l'atmosphère par faible ICU}

- les cartes au sol et en altitude (surface $700 \mathrm{hPa}$ ) sont issues du Bulletin Météorologique Européen (BME) : la lettre H (high) correspond aux anticyclones (carte au sol) et aux hauts géopotentiels (carte d'altitude), la lettre L (low) aux dépressions et bas géopotentiels ;

- les informations météorologiques du jour sont fournies par Météo-France ; 
- les profils verticaux dans les basses couches de l'atmosphère proviennent des sondages effectués par Météo-France mais archivés par le site du Department of Atmospheric Science de l'Université du Wyoming (adresse du site internet en fin de bibliographie).

\section{Situation « classique » : exemple du 18 décembre 1999 (fig. 17)}

Fig. 17 : Situation « classique » par faible îlot de chaleur urbain (perturbation d'ouest)

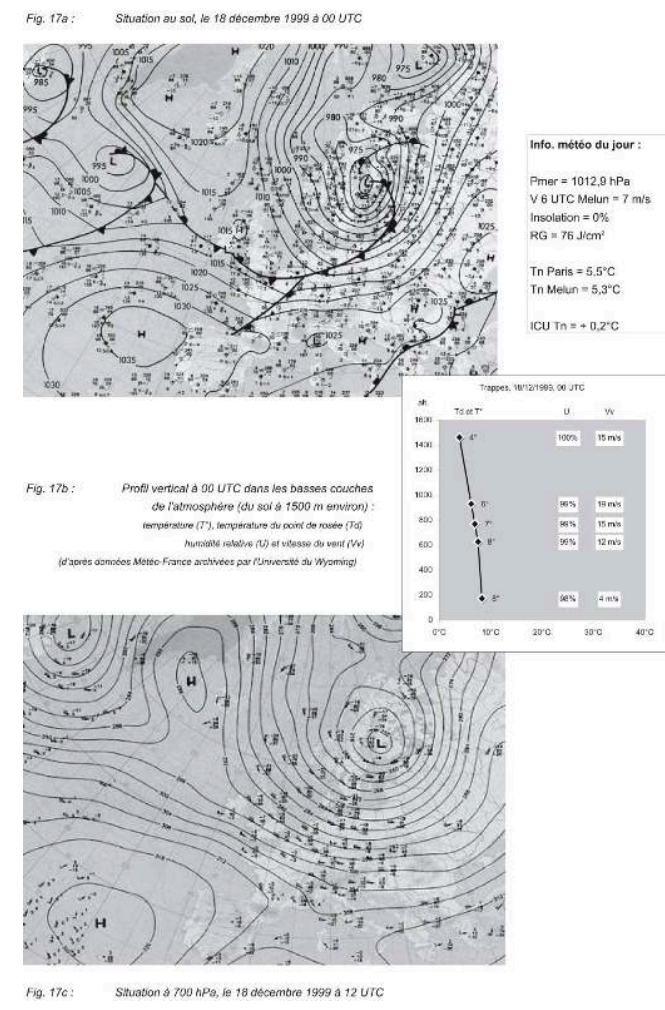

En ce 18 décembre 1999, l'ICU a pratiquement disparu $\left(+0,2^{\circ} \mathrm{C}\right)$. Il s'agit d'un exemple typique de situation cyclonique perturbée. Entre les très bas géopotentiels centrés sur la Mer Baltique et les hauts géopotentiels des Açores (fig. 17c), on observe une circulation d'ouest à nord-ouest rapide où se succèdent des fronts (fig. 17a). Nous sommes dans le secteur chaud de la perturbation en présence d'une masse d'air totalement saturé dans les basses couches et d'un vent rapide au sol et plus encore en altitude (fig. 17b).

Le ciel totalement couvert (fraction d'insolation nulle) et la force du vent au sol $(7 \mathrm{~m} / \mathrm{s}$ à Melun à 6:00 UTC et $4 \mathrm{~m} / \mathrm{s}$ à Trappes à 0:00 UTC) empêchent alors la formation de l'ICU. Cette situation est très fréquente en hiver, période préférentielle des flux d'ouest perturbés, en relation avec la balance saisonnière des centres d'action barométrique qui amènent plus ou moins directement à nos latitudes des masses d'air polaire maritime frais et humide. La persistance de ces types de temps nébuleux et venteux sur parfois plusieurs semaines consécutives explique la très grande majorité $(56 \%)$ des très faibles ICU décadaires pour cette seule saison (fig. $6 \mathrm{~b}$ ).

Situation « atypique » : exemple du 21 décembre 1999 (fig. 18) 
Fig. 18 : Situation « atypique » par faible îlot de chaleur urbain (hautes pressions)

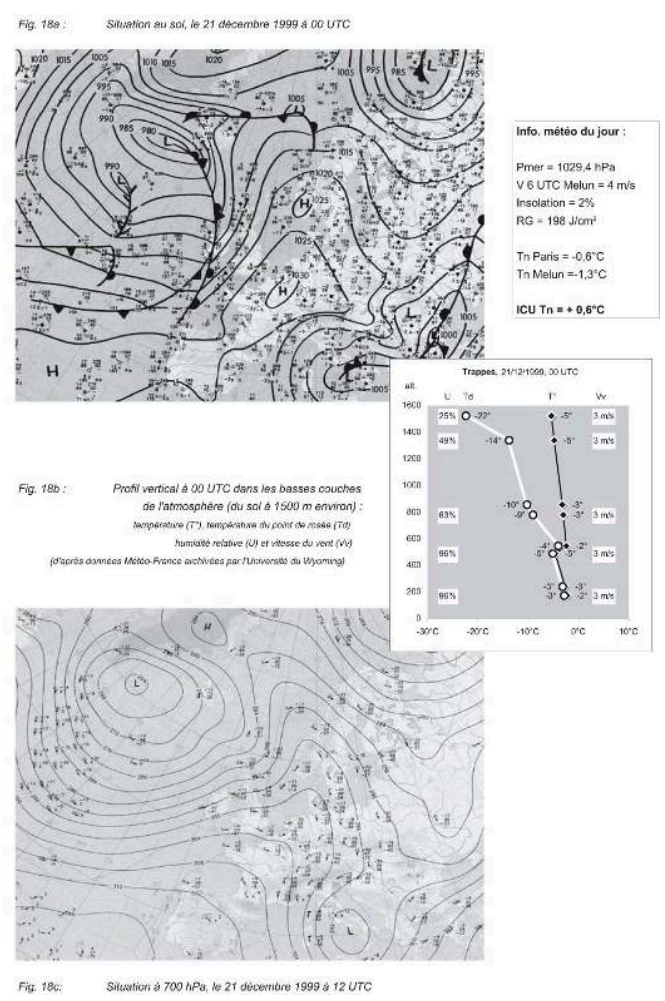

50 Trois jours plus tard, l'ICU est presque toujours aussi peu marqué $\left(+0,6^{\circ} \mathrm{C}\right)$, pourtant la situation synoptique paraissait a priori beaucoup plus propice à sa formation.

Au sol comme en altitude (fig. 18a et 18c), les basses pressions se sont éloignées vers le nord-est de la Scandinavie, en mer de Barents, tandis que les hautes pressions d'origine subtropicale se prolongeaient vers l'Europe occidentale jusqu'à la Scandinavie sous forme d'une dorsale et même d'une petite cellule anticyclonique de $1030 \mathrm{hPa}$ centrée sur le Luxembourg. En région parisienne, malgré ces hautes pressions $(1029,4 \mathrm{hPa})$, si le vent est faible, en revanche le ciel reste couvert (fraction d'insolation $=2 \%$ ). Le radiosondage de Trappes montre une quasi saturation de l'air dans les cinq cents premiers mètres de l'atmosphère (96\%), alors qu'au-dessus le temps est clair, sec (25\%) et relativement calme $(3 \mathrm{~m} / \mathrm{s})$ " conformément » au contexte barométrique (fig. 18b). A cette saison, l'humidité du sol et le refroidissement créent fréquemment une forte nébulosité dans les basses couches: une seule des deux conditions sine qua non étant donc remplie (air calme), la formation de l'ICU est donc neutralisée... Ainsi les périodes anticycloniques d'automne et d'hiver, qui constituent des ruptures temporaires dans la succession des perturbations d'ouest, n'offrent-elles pas souvent des forts ICU. Des cas de très forts ICU se produisent quelquefois en hiver dans les masses d'air continental sec établis depuis plusieurs jours, notamment lorsqu'une couche de neige " héritée » persiste au sol sur la campagne et entraîne des inversions thermiques nocturnes puissantes. En ville, le déneigement artificiel limite alors le refroidissement par rayonnement et l'écart avec la campagne est parfois supérieur à $10^{\circ} \mathrm{C}$ (Cantat, 1989). 


\title{
Situation synoptique et profil vertical de l'atmosphère par fort ICU
}

\author{
Situation « classique » : exemple du $1^{\mathrm{er}}$ août 1999 (fig. 19)
}

Fig. 19 : Situation « classique » par fort îlot de chaleur urbain (hautes pressions)

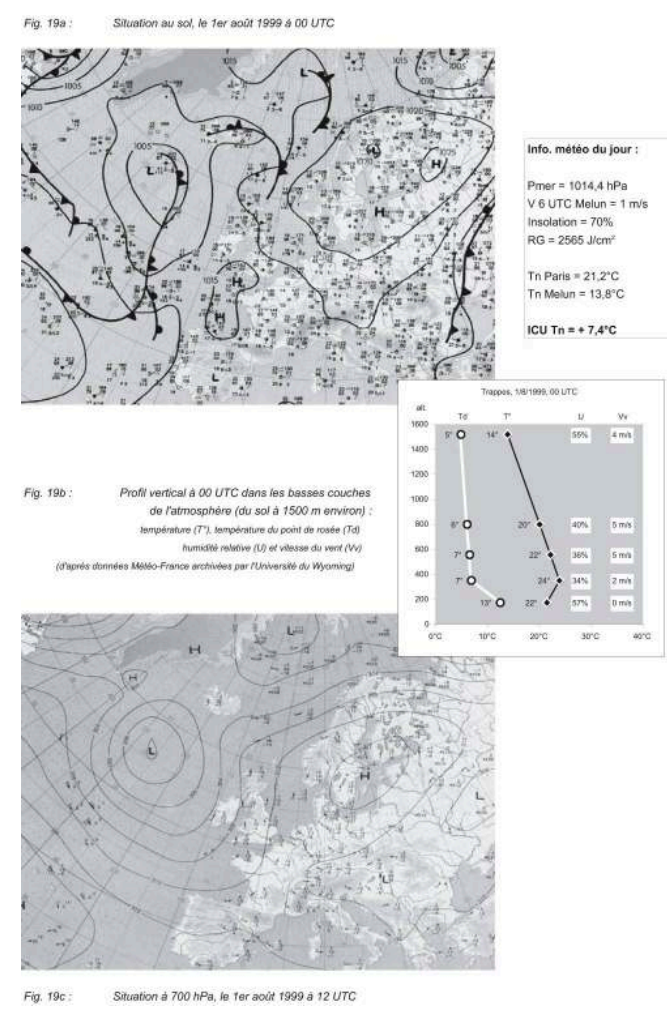

52 Le $1^{\text {er }}$ août 1999 , un temps clair et calme règne sur pratiquement toute l'Europe, en relation avec des hautes pressions dynamiques centrées sur la Mer Baltique qui rejettent sur l'Atlantique et l'Océan Arctique les perturbations océaniques (fig. 19a et 19c). Cette situation illustre le cas le plus fréquent des forts ICU, avec un renforcement possible de son intensité dû à un emmagasinement calorifique durant plusieurs jours consécutifs de "beau temps" (effet cumulatif).

Si la pression au sol à Paris n'est pas très élevée $(1014,4 \mathrm{hPa})$, ce qui est fréquent en été, les cartes nous montrent toutefois une très nette influence anticyclonique. L'espacement des isobares occasionne une absence totale de vent $(0 \mathrm{~m} / \mathrm{s}$ à Trappes et Melun). La sécheresse de l'air dans toute la colonne d'atmosphère (entre 34 et $57 \%$ dans les 1500 premiers mètres, fig. 19b) favorise un fort ensoleillement. Nous sommes en fait dans une période chaude et sèche qui dure depuis environ une semaine. La chaleur accumulée dans la journée par les matériaux urbains depuis plusieurs jours limite le refroidissement nocturne dans Paris : les valeurs minimales restent supérieures à $20^{\circ} \mathrm{C}$ $\left(21,2^{\circ} \mathrm{C}\right.$ à St-Jacques), contre 11 à $15^{\circ} \mathrm{C}$ sur la campagne $\left(13,8^{\circ} \mathrm{C}\right.$ à Melun). Par ailleurs, nous observons déjà à 0:00 UTC le début de formation d'une légère inversion de température, phénomène observé systématiquement lors des ICU marqués.

En vue de la modélisation de l'ICU, un approfondissement de la connaissance des effets d'accumulation serait souhaitable. Cette accumulation devrait être envisagée à l'échelle de l'épisode de temps clair et calme (de quelques jours à une décade environ), mais aussi comme nous l'avons mentionné précédemment à l'échelle saisonnière (cf. 
comportement atypique du début d'automne et « retard» tardi-printanier et estival fréquent).

Situation « atypique » : exemple du 6 août 1999 (fig. 20)

Fig. 20 : Situation « atypique » par fort îlot de chaleur urbain (marais barométrique)

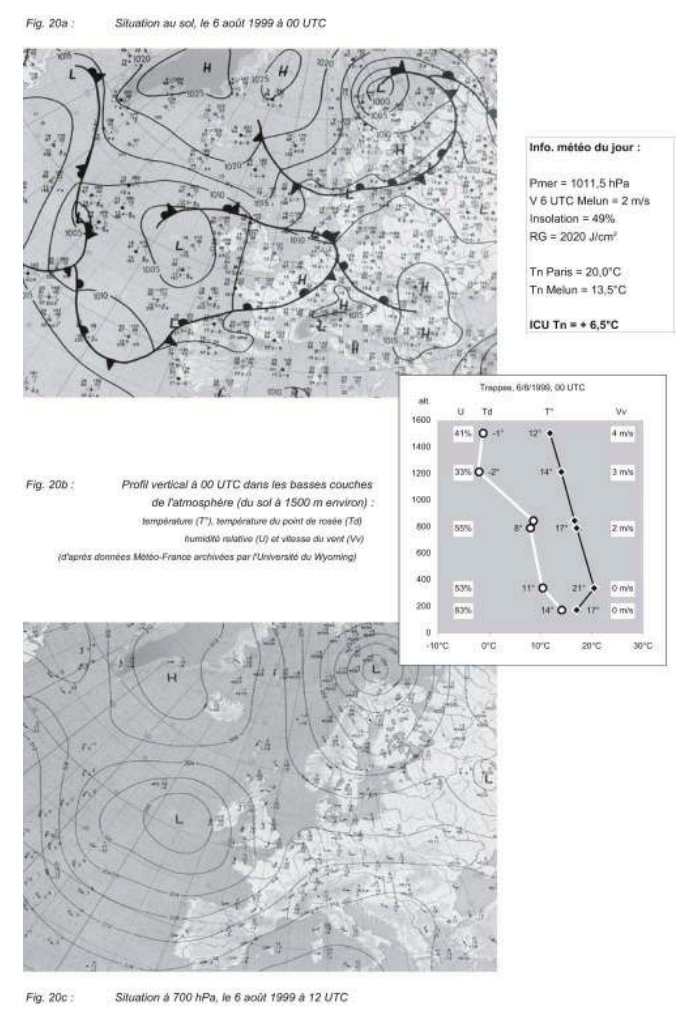

55 Le 6 août 1999, la configuration barométrique a totalement changé : en altitude, les hauts géopotentiels scandinaves qui "protégeaient » l'Europe occidentale ont disparu alors que les bas géopotentiels océaniques ont migré du centre de l'Atlantique vers l'Irlande. Au sol, la situation est plus confuse: des fronts parcourent en tous sens l'océan et le continent. À 6:00 UTC, la région parisienne se trouve prise dans un flux de sud, entre un front occlus qui s'évacue lentement vers le nord de l'Angleterre et un front chaud qui remonte progressivement de la vallée de la Loire. À Paris, la pression au sol est relativement basse $(1011,5 \mathrm{hPa})$, un marais barométrique affecte une grande partie de l'Europe. Si ce n'est le vent faible (entre 0 et $1 \mathrm{~m} / \mathrm{s}$ au sol et dans les très basses couches), les conditions ne semblent guère propices à la formation d'un ICU...

L'ICU de $+6,5^{\circ} \mathrm{C}$ parait dans ces conditions remarquablement élevé. Il s'explique en fait par la conjugaison de trois phénomènes :

- à cette saison, entre les passages frontaux la puissance du rayonnement solaire peut assécher l'atmosphère et occasionner de longues plages de ciel clair (49\% d'insolation) ;

- le dégagement de l'air est favorisé ici par un phénomène de divergence-subsidence en altitude (fig. 20c) qui se marque par un air sec en altitude (fig. 20b). Cette origine dynamique permet aux éclaircies de se produire durant la nuit. Associées au vent calme, la mise en place des mécanismes de l'ICU est possible ;

- le temps plus perturbé qui se met en place sur l'Europe occidentale a été précédé par une longue période de beau temps (cf. 1er août) où la chaleur solaire a pu s'accumuler dans les 
matériaux. Ainsi, à la faveur d'une éclaircie nocturne le différentiel ville/campagne est

encore très marqué.

traduisent la nécessité d'envisager l'atmosphère comme un tout indivisible, les phénomènes de basses couches qui nous intéressent tout particulièrement dépendant étroitement de la structure verticale de l'atmosphère dans son ensemble. Pour mieux saisir encore l'ICU dans sa forme et sa genèse, il serait également souhaitable de mieux connaître l'aérologie et la température des très basses couches (relevés de la Tour Eiffel par exemple et autres...).

\section{Conclusion}

\section{De l'observation à la prévision...}

La diversité des configurations de l'îlot de chaleur urbain repose donc sur la complexité des interrelations entre :

- des données géographiques stables (situation, site, type d'urbanisation...)

- des données végétales et atmosphériques déterminées par des événements cycliques (saisons)

- des données atmosphériques aléatoires (types de circulation et types de temps)

En région de climat tempéré océanique, la très forte variabilité décadaire, et surtout journalière, de l'îlot de chaleur urbain peut donc être considérée comme un révélateur des types de temps dans la mesure où les autres facteurs dont dépend cette anomalie thermique sont constants (facteurs spatiaux) ou à évolution lente et régulière (facteurs saisonniers).

Pour prédire un fort ICU, il convient de prévoir un temps clair et calme... Ce type de temps s'observe généralement dans les périodes anticycloniques, ce qui relève de la circulation atmosphérique générale. Or, si les centres météorologiques sont capables de prévoir avec une assez grande fiabilité la situation synoptique à courte et moyenne échéance, en revanche la prévision des types de temps est plus délicate car ils s'observent à des échelles parfois plus fines que la maille des modèles de prévision. Dans le plan vertical, il arrive fréquemment que des phénomènes de basses couches viennent «brouiller » la qualité globale d'une prévision. Quand il s'agit de nuages bas, cela remet alors totalement en cause les mécanismes de formation de l'ICU en bloquant le refroidissement nocturne par rayonnement. La connaissance du terrain des prévisionnistes est alors essentielle pour interpréter les sorties de modèles et adapter les prévisions à l'échelle locale, en fonction de facteurs géographiques comme la présence d'une grande agglomération.

61 En y intégrant les spécificités de la ville, les modèles de prévision météorologiques d'échelle synoptique pourraient permettre de prévoir l'intensité de l'ICU. Cette connaissance s'avérerait utile, par exemple, pour anticiper les épisodes de forte pollution atmosphérique au centre de l'agglomération lorsque par temps calme l'excédent thermique provoque un phénomène de brise de campagne: "La convergence de ces vents faibles ( 1 à $3 \mathrm{~m} / \mathrm{s}$ ) vers le point le plus chaud, provoque, dans cet endroit, une accumulation de polluants d'autant plus importante que la brise représente une couche d'inversion d' 1 à $3^{\circ}$ plus fraîche, favorable à une concentration 
des polluants près du sol » (Escourrou 1991). Si la convergence produit nécessairement une ascendance au centre de l'ICU, celle-ci n'est pas assez puissante pour évacuer les polluants lourds dans l'atmosphère. En présence d'un type de temps clair et calme, la détermination de ces quartiers à risques se heurte à une absence de connaissance à échelle très fine de l'îlot de chaleur urbain qui permettrait de mieux connaître les lieux de convergence et de blocage des masses d'air polluées. En effet, la diversité thermique réelle de Paris ne peut être traduite par quelques mesures ponctuelles ( 3 ou 4 actuellement) et dans des sites normalisés (environnement végétal et relativement dégagé) peu révélateurs des «ambiances » urbaines. Des mesures effectuées avec le centre météorologique de Montsouris ont montré qu'en hiver la partie la plus chaude de l'agglomération était située dans le VIII ${ }^{\mathrm{e}}$ arrondissement ( $2^{\circ}$ de plus qu'à St-Jacques). En été, la Seine apparaît comme une partie plus fraîche, les températures augmentant alors vers le sud et surtout vers le nord avec un maximum dans le $\mathrm{X}^{\mathrm{e}}$ arrondissement (Escourrou 1988).

62 La spatialisation de détail de l'îlot de chaleur parisien pourrait être réalisée grâce à l'utilisation d'un Système d'Information Géographique (S.I.G.) croisant des informations relatives à l'espace (géométrie du bâti, types de surface) et des mesures représentatives de la diversité thermique réelle des sites urbains (à l'instar des travaux de Charabi en 2001 sur la métropole lilloise, par exemple). Dans un souci de climatologie appliquée (pollution, santé...), il pourrait être intéressant d'inclure à ces modèles statiques la mobilité spatiale et temporelle de l'ICU. En effet, le vent est à l'origine de déformations dont ne peuvent rendre compte les seules caractéristiques physiques de la ville (exportation de chaleur urbaine sur la campagne et/ou importation de fraîcheur rurale dans l'agglomération). La compréhension globale de l'îlot de chaleur urbain est un préalable à sa modélisation et a fortiori sa prévision. L'influence capitale des types de temps dans la genèse de l'ICU implique une approche tridimensionnelle et dynamique de l'atmosphère.

63 Ces réflexions rappellent l'importance des emboitements et interrelations entre les différentes échelles spatio-temporelles dans la connaissance d'un phénomène discontinu et mouvant.

\section{BIBLIOGRAPHIE}

BARROT C., 1970 : Incidences de l'îlot de chaleur parisien sur les aérodromes d'Orly et du Bourget : modifications locales des conditions météorologiques. Mémorial Météorologie Nationale.

BASTIE, J., 1984 : Géographie du Grand Paris. Masson, 208 p.

BESSEMOULIN P., 1980 : « Urbanisation et Météorologie », La Météorologie, n² 23, pp. 51-67.

BESSON L., 1921 : « L'influence de Paris sur la radiation reçue du Soleil, et dans la région environnante », ASTHVP, t. 10, 16 p. 
BORNSTEIN R., 1968 : « Observations of the Urban Heat Island Effect in New-York City », Journal of Applied Meteorology, $\mathrm{n}^{\circ} 7$, pp. 575-582.

BRAZIER C., PERDEREAU L., 1931 : Exemple d'une altération du climat, résultat d'une activité humaine : le brouillard à St-Maur de 1874 à nos jours. Météorologie Nationale.

-, 1980 : «Les fortes intensités de précipitations à Paris », La Météorologie, n 20-21, pp. 99-104.

CALVET C., 1984 : Climatologie de la région parisienne. Météorologie Nationale, Données et statistiques, $\mathrm{n}^{\circ} 5,61 \mathrm{p}$.

CANTAT O., 1986 : « Influence de l'urbanisation sur le climat de l'agglomération parisienne », Physio-Géo, n 16, pp. 25-40.

-, 1987 : Télédétection spatiale et microclimats : le cas de la région d'Ile-de-France. Institut d'Aménagement et d'Urbanisme de la Région Ile-de-France (IAURIF), 190 p.

-,1988a : L'incidence des gradients thermiques de surface sur les précipitations en Ile-de-France. Les apports de la télédétection spatiale. Physio-Géo, n 19, pp. 15-25.

,$- 1988 \mathrm{~b}$ : La radiation solaire et l'organisation urbaine. Cahiers du CREPIF, n²2, pp. 20-30.

-, 1989 : Contribution à l'étude des variations du bilan d'énergie en région parisienne. Essai sur les bilans d'énergie dans les grandes métropoles. Thèse de doctorat, Université de Paris IV-Sorbonne, 2 volumes, $362 \mathrm{p}$. et $254 \mathrm{p}$.

-, 1993 : Conséquences climatiques des variations du bilan d'énergie en région parisienne. Géographie Physique et Environnement, $\mathrm{n}^{\circ}$ 1, Presses Universitaires de Caen , pp. 19-36.

-, BRUNET L., 2001 : Discontinuité géographique et particularités climatiques en Basse-Normandie. Annales de Géographie, ${ }^{\circ}$ 622, pp. 579-596.

-, BRUNET L., 2003 : « L'originalité bioclimatique de l'escarpement rocheux de Rouvrou (Suisse normande, France) », Sécheresse, vol. 14, n 1, pp. 15-22.

CARREGA P., 1994 : Topoclimatologie et Habitat. Thèse de Doctorat, Revue de géographie du Laboratoire d'analyse spatiale Raoul Bachelard, n³5 et 36, Université Sophia Antipolis de Nice, $408 \mathrm{p}$.

CHANDLER T., 1965 : The Climate of London. Hutchinson \& Co, 292 p.

-, 1970 : Urban climatology. Inventory and prospect. WMO, Technical Note, $\mathrm{n}^{\circ} 108, \mathrm{pp} .1-14$.

CHARABI Y., 2000 : L'îlot de chaleur urbain de la métropole lilloise : mesures et spatialisation. Thèse de Doctorat de l'Université des Sciences et Technologies de Lille, $247 \mathrm{p}$.

-, BIGOT S., KERGOMARD C., 2002 : Interpolation et cartographie automatique de la température nocturne en milieu urbain. Publication de l'AIC, vol. 14, pp. 271-278.

DETTWILLER J., 1970 : Evolution séculaire du climat de Paris. Influence de l'urbanisation. Mémorial Météorologie Nationale, $\mathrm{n}^{\circ} 52,83 \mathrm{p}$.

DURAND-DASTES F., 1973 : A propos des notions de types de temps et de type de circulation. Note rédigée à la suite des discussions de la commission de climatologie, $\mathrm{CNG}, 4 \mathrm{p}$.

ESCOURROU G., 1980 : «L'influence de l'urbanisation sur les précipitations de la région parisienne », Cahiers de géographie Physique, n4, Université de Lille, pp. 55-66.

-, 1983 : «L'évolution des brouillards dans la région parisienne », Hommes et Terres du Nord, Lille, $\mathrm{n}^{\circ} 3$, pp. 60-64. 
_, 1986a : « Le climat de l'agglomération parisienne », L’Information Géographique, n50, pp. 96-102.

,$- 1986 \mathrm{~b}$ : « Les brises de campagne : un aspect essentiel du climat urbain », International Symposium on Urban and Local Climatology (Freiburg), pp. 87-95.

-, 1988 : «Les problèmes posés par le climat parisien », Cahiers du CREPIF, n²2, pp. 10-19.

-, 1990 : « La spécificité du climat de la région parisienne », Revue de Géographie de Lyon, n65, pp. 85-89.

-, 1991 : Le climat et la ville. Nathan Université, 192 p.

-, 1988 : «Le climat et la santé de l'homme en Ile-de-France », Les Cahiers du CREPIF, n²2, pp. 31-43.

GALLO C., 1988 : Les brises de campagne à Paris. Thèse de doctorat de 3e cycle, Université de ParisSorbonne, $266 \mathrm{p}$.

GRISOLLET H., 1958 : Climatologie de Paris et de la région parisienne. Mémorial Météorologie Nationale, $\mathrm{n}^{\circ} 45,78 \mathrm{p}$.

-, 1965 : Etude du vent et de la brise diurne à Paris au sommet de la Tour-St-Jacques. Monographie Météorologie Nationale, $\mathrm{n}^{\circ} 46,30 \mathrm{p}$.

JANICOT S., 1984 : Etude climatologique des brouillards à Orly. Note Technique, Météorologie Nationale, $n^{\circ} 10$.

LANDSBERG H, 1970 : « Micrometeorological temperature differentiation through urbanization », WMO, Technical Note, ${ }^{\circ} 108$, pp. 129-136.

LE GUISCHER Y., TSCHIRHARRT G., 1981 : Etude climatologique du brouillard à Orly. Note Technique, Météorologie Nationale, $19 \mathrm{p}$.

LEROY M., 1999 : Classification d'un site. Note technique n³5. Direction des systèmes d'observation, Météo France, $12 \mathrm{p}$.

,- 2002 : «La mesure au sol de la température et des précipitations », La Météorologie, n³9, pp.

$52-56$.

MAURAIN C., 1947 : Le climat de Paris. PUF, 164 p.

OKE T.R., 1973 : «City size and urban heat island », Atmospheric Environment, Oxford, England, $\mathrm{n}^{\circ} 7$, pp. 769-779.

PEDELABORDE P., 1958 : Le climat du bassin parisien : essai d'une méthode rationnelle de climatologie physique. Editions M.-Th. Génin, 2 volumes, 539 et 116 p.

PEDELABORDE P., 1970 : Introduction à l'étude scientifique du climat. SEDES, 246 p.

PERRIN DE BRICHAMBAUT C., LEROY M., 1995 : La mesure de la température de l'air. La Météorologie, $\mathrm{n}^{\circ} 12$, pp. $14-30$.

PIERY M. et al, 1934 : Traité de climatologie biologique et médicale. Chapitre éléments météorologiques du climat « Les éléments météorologiques du climat ». Paris, 3 volumes, 2664 p.

REQUILLARD G., 1961 : De l'influence thermique de Paris et de ses répercussions sur la banlieue proche. Météorologie Nationale (manuscrit consultable à la bibliothèque de Météo-France).

YosHINo M. M., 1975 : Climate in a small area. University of Tokyo Press, 549 p. 
http://weather.uwyo.edu/upperair/sounding.html[données gratuites de l'University of Wyoming (Department of Atmospheric Science)]

\section{RÉSUMÉS}

L'apparition d'un îlot de chaleur urbain (ICU) est la manifestation climatique la plus concrète de la présence de l'agglomération parisienne, avec un excédent moyen supérieur à $3^{\circ} \mathrm{C}$ pour les valeurs nocturnes, disparaissant presque totalement dans la journée. L'analyse des températures minimales est de ce fait en grande partie représentative de l'ICU. Son intensité est plus marquée en été $\left(3,4^{\circ} \mathrm{C}\right)$ qu'en hiver $\left(2,5^{\circ} \mathrm{C}\right)$ mais, au-delà des moyennes, elle varie beaucoup d'un jour à l'autre : selon les types de temps, les écarts ville/campagne peuvent passer de 0 à plus de $10^{\circ} \mathrm{C}$. La densité actuelle du réseau de mesures ( 55 postes sur $10.000 \mathrm{~km}^{2}$ ) précise les caractéristiques de forme et d'étendue de cette anomalie thermique. Une analyse statistique souligne l'influence essentielle de la nébulosité et du vent sur la formation de l'ICU, les autres paramètres atmosphériques apportant une information en grande partie redondante et donc non discriminante. L'association de ces deux composantes permet alors d'identifier un type de temps préférentiel (clair et calme) et de proposer une approche de climatologie dynamique nécessaire à la modélisation et à la prévision du phénomène. Les périodes anticycloniques et de circulations lentes semblent logiquement les plus propices à la formation d'un ICU marqué, mais pas uniquement. Pour expliquer des cas "non-conformes ", les relations entre situation synoptique, types de circulation, types de temps et ICU sont précisées ici. Les radiosondages ont permis de mieux appréhender les mécanismes de formation de l'ICU grâce à la mise en évidence notamment de combinaisons atmosphériques génératrices de couvertures nuageuses ou d'éclaircies.

The observation of an Urban Heat Island (UHI) is the more concrete demonstration of the climatic effect of the urbanized area of Paris. The city of Paris records a mean thermal positive anomaly of $3^{\circ} \mathrm{C}$ during the night, which almost totally vanishes during the day. The nocturnal intensity of the UHI varies from day to day, depending on the weather types: the urban-rural thermal difference can vary between 0 and more than $10^{\circ} \mathrm{C}$. The present density of the meteorological grid system measurements (55 stations / $10.000 \mathrm{~km}^{2}$ ) allows to describe form and surface of this thermal anomaly. Results of a statistical analysis underlined the essential influence of cloudiness and wind, but the other climatic elements cannot significantly explain the development of the UHI. The cloudiness-wind association allows to identify a preferential weather type (clear and calm) and to propose a dynamic climatic approach for the UHI development modelling. The meteorological situations with a high surface atmospheric pressure and a light prevailing wind are more favourable to the UHI development although there are some exceptions... The measurements at different levels (temperature, dew point, wind and humidity) allow to explain the atmospheric features inducing overcast sky or breaks.

\section{INDEX}

Index géographique : France, Paris

Mots-clés : climatologie urbaine, îlot de chaleur, radiosondages, type de temps

Keywords : meteorological network, urban climatology, urban heat island, weather type 


\section{AUTEUR}

\section{OLIVIER CANTAT}

Laboratoire GEOPHEN (LETG - UMR 6554 CNRS), Université de Caen Basse-Normandie, Esplanade de la Paix, BP 5186 - 14032 Caen cedex, cantat@geo.unicaen.fr 